\title{
Modification of aluminum alkoxides with $\beta$-ketoesters: new insights into formation, structure and stability
}

\author{
Robert Lichtenberger · Michael Puchberger • \\ Stefan O. Baumann • Ulrich Schubert
}

Received: 7 July 2008/Accepted: 29 December 2008/Published online: 21 January 2009

(C) The Author(s) 2009. This article is published with open access at Springerlink.com

\begin{abstract}
Al}\left(\mathrm{O}^{\mathrm{i}} \mathrm{Pr}\right)_{2}(\beta \text {-ketoesterate })\right]_{2}$ and $\mathrm{Al}(\beta$-ketoesterate $)_{3}$ ( $\beta$-ketoesterate $=$ methyl, ethyl, iso-propyl, tertbutyl, allyl and 2-(methacryloyloxy)ethyl acetoacetate) were prepared by reaction of $\left[\mathrm{Al}\left(\mathrm{O}^{\mathrm{i}} \mathrm{Pr}\right)_{3}\right]_{4}$ with the corresponding $\beta$-ketoesters. $\operatorname{Al}(\beta \text {-ketoesterate })_{3}$ derivatives were exclusively formed at room temperature, whereas elevated reaction temperatures, causing thermal de-oligomerization of $\left[\mathrm{Al}\left(\mathrm{O}^{\mathrm{i}} \mathrm{Pr}\right)_{3}\right]_{4}$, were necessary for the formation of $\left[\mathrm{Al}\left(\mathrm{O}^{\mathrm{i}} \mathrm{Pr}\right)_{2}(\beta \text {-ketoesterate })\right]_{2}$. All compounds were characterized by NMR spectroscopy, and $\left[\mathrm{Al}\left(\mathrm{O}^{\mathrm{i}} \mathrm{Pr}\right)_{2}\right.$ (tert-butyl acetoacetate) $]_{2}$ by a single crystal structure analysis. The $\left[\mathrm{Al}\left(\mathrm{O}^{\mathrm{i}} \mathrm{Pr}\right)_{2}(\beta \text {-ketoesterate })\right]_{2}$ derivatives are asymmetrically substituted dimers with one octahedrally and one tetrahedrally substituted aluminum atom, bridged by two isopropoxo groups, whereas the $\mathrm{Al}(\beta \text {-ketoesterate })_{3}$ derivatives are monomers with octahedrally coordinated aluminum. Transesterification as a possible side reaction was only observed at elevated temperatures for $\mathrm{Al}$ (tert-butyl acetoacetate $)_{3}$ in the presence of liberated iso-propanol.
\end{abstract}

Keywords Aluminum alkoxide derivatives . $\beta$-Ketoesterates - Transesterification - Ligand exchange

Dedicated to David Avnir on the occasion of his 60th birthday.

R. Lichtenberger - M. Puchberger · S. O. Baumann ·

U. Schubert $(\bowtie)$

Institute of Materials Chemistry, Vienna University

of Technology, Getreidemarkt 9, 1060 Wien, Austria

e-mail: uschuber@mail.zserv.tuwien.ac.at

\section{Introduction}

One problem in the sol-gel chemistry of metal alkoxides is their high reaction rates during hydrolysis and condensation, compared to silicon alkoxides, and the resulting tendency to form precipitates instead of gels. Lowering of their reactivity can be achieved by substitution of one or more alkoxo groups by bi- or even multidentate ligands which are bound more strongly to the metal as a consequence of the chelate effect. Since the number of hydrolysable alkoxo groups is thus reduced and additional coordination sites at the metal centers are blocked, the rate of hydrolysis and the degree of cross-linking in the formed network are lowered [1].

Another consequence of the higher Lewis acidity of metal alkoxides is their tendency to form oligomers $\left[\mathrm{M}(\mathrm{OR})_{x}\right]_{n}$ via alkoxo bridges. The chemical and physical properties of the alkoxides strongly depend on the degree of oligomerization, which in turn is a result of the electronic and steric properties of the metal center and the alkoxo group. For example, aluminum iso-propoxide is tetrameric $\left[\mathrm{Al}\left(\mathrm{O}^{\mathrm{i}} \mathrm{Pr}\right)_{3}\right]_{4}[2,3]$, whereas the tert-butoxide $\left[\mathrm{Al}\left(\mathrm{O}^{\mathrm{t}} \mathrm{Bu}\right)_{3}\right]_{2}$ is dimeric [4] in the solid state as well as in solution at room temperature. This example shows the inverse correlation between steric demand of the alkoxo group and degree of oligomerization. This tendency of metal alkoxides to oligomerize is retained after modification with bidentate organic ligands. Thus, aluminum alkoxides and siloxides are known to form mono-, di-, tri-, or oligomeric species upon modification with $\beta$-diketones [5, 6].

Modification of metal alkoxides with bidentate organic ligands also opens the possibility to introduce additional functionalities by using ligands bearing a functional organic group, e.g. polymerizable groups for the formation of class II inorganic-organic hybrid polymers. As a matter 


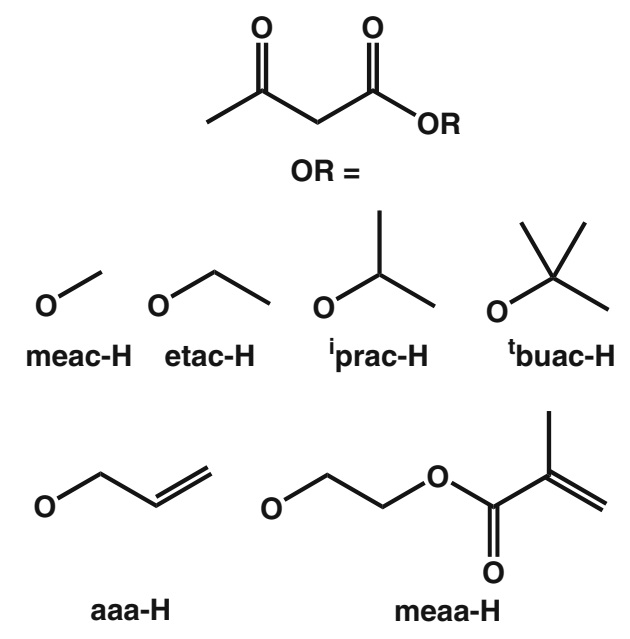

Fig. $1 \beta$-Ketoesters used for modification of $\mathrm{Al}\left(\mathrm{O}^{\mathrm{i}} \mathrm{Pr}\right)_{3}$

of fact, allyl (aaa-H) and 2-(methacryloyloxy)ethyl acetoacetate (meaa-H) (see Fig. 1) have often been used in solgel chemistry for this purpose for various metal alkoxides. Own previous work was mainly focused on the modification and functionalization of titanium and zirconium alkoxides by nitrogen-containing ligands [7]. In this work, we expand this work on aluminum alkoxides modified by reaction with $\beta$-ketoesters. Whereas the influence of the alkoxo or siloxo group on the oligomerization and structure of acac-substituted (acac $=$ acetylacetonate) aluminum alkoxides or siloxides was studied in detail [5], this is not the case for variations of the ester OR groups in $\beta$-ketoesterate derivatives $(\beta$-ketoesterate $=$ anion of $\beta$-ketoester). Since the bonding of $\beta$-ketoesterate ligands to the metal center is inherently asymmetrical (contrary to $\beta$-diketonate derivatives), because of the different electronic influence of the alkyl and OR substituents at the carbonyl carbon atoms, differences can be anticipated. In this article we report the results of our investigations on the formation and stability of aluminum iso-propoxide derivatives modified by methyl (meac-H), ethyl (etac-H), iso-propyl ('prac$\mathrm{H})$ and tert-butyl ('buac- $\mathrm{H})$ acetoacetate as model ligands, as well as allyl (aaa-H) and 2-(methacryloyloxy)ethyl (meaa-H) acetoacetate as ligands with polymerizable groups (Fig. 1), including investigation of transesterification as possible side reaction. The influence of variation the ester group is reported as well as differences in coordination behavior of $\beta$-ketoesters compared to $\beta$-diketones.

\section{Experimental}

All operations were carried out in a moisture and oxygen free atmosphere of dry argon using standard Schlenk or glove box techniques. $\mathrm{Al}\left(\mathrm{O}^{\mathrm{i}} \mathrm{Pr}\right)_{3}$ (Aldrich, $\left.98+\%\right)$, methyl acetoacetate (Aldrich, 99\%) ethyl acetoacetate (Fluka, p.a.), iso-propyl acetoacetate (Alfa Aesar, 98\%), tert-butyl acetoacetate (Aldrich, 98\%), allyl acetoacetate (Aldrich, 98\%) and 2-(methacryloyloxy)ethyl acetoacetate (Aldrich, 95\%) were used as received. All solvents were dried and purified by standard techniques. $\mathrm{C}_{6} \mathrm{D}_{6}(99.5 \%$, euriso-top) and $\mathrm{d}_{8}$-toluene ( $99.6 \%$, euriso-top) used for NMR experiments were dried over $3 \AA$ molecular sieve and degassed. $1 \mathrm{D}{ }^{1} \mathrm{H}$ and ${ }^{13} \mathrm{C}$ NMR spectra were recorded on a Bruker AVANCE $250\left(250.13 \mathrm{MHz}\left\{{ }^{1} \mathrm{H}\right\}, 62.86 \mathrm{MHz}\left\{{ }^{13} \mathrm{C}\right\}\right)$ spectrometer. ${ }^{27} \mathrm{Al}$ and $2 \mathrm{D}$ NMR spectra were recorded on a Bruker AVANCE $300\left(300.13 \mathrm{MHz}\left\{{ }^{1} \mathrm{H}\right\}, 75.47 \mathrm{MHz}\right.$ $\left.\left\{{ }^{13} \mathrm{C}\right\}, 78.21 \mathrm{MHz}\left\{{ }^{27} \mathrm{Al}\right\}\right)$ spectrometer. Both spectrometers were equipped with a $5 \mathrm{~mm}$ broadband probe head and a z-gradient unit. COSY (Correlated Spectroscopy), HSQC (Heteronuclear Single Quantum Correlation), HMBC (Heteronuclear Multiple-Bond Correlation, evolution delay for long range coupling $100 \mathrm{~ms}$ ), and EXSY (Exchange Spectroscopy, $t_{\text {mix }}=1.2 \mathrm{~s}$ ) were measured with Bruker standard pulse sequences. The ${ }^{27} \mathrm{Al}$ NMR signals were referenced externally against a $2 \mathrm{M}$ solution of $\mathrm{AlCl}_{3}$ in water $(0 \mathrm{ppm})$.

\section{$2.1\left[\mathrm{Al}\left(\mathrm{O}^{\mathrm{i}} \mathrm{Pr}\right)_{2}(\mathrm{meac})\right]_{2}$}

An amount of $1.003 \mathrm{~g}(4.91 \mathrm{mmol})$ of $\mathrm{Al}\left(\mathrm{O}^{\mathrm{i}} \mathrm{Pr}\right)_{3}$ was dissolved in $10 \mathrm{~mL}$ of toluene at room temperature followed by slow addition of $0.570 \mathrm{~g}(4.91 \mathrm{mmol})$ of methyl acetoacetate under stirring. The clear reaction solution was stirred at $120{ }^{\circ} \mathrm{C}$ for $18 \mathrm{~h}$. The volatiles were then removed in vacuo. The crude product was washed with $2 \mathrm{~mL}$ of n-pentane and a colorless oil obtained. Yield $1.217 \mathrm{~g} \mathrm{(95 \% ).}$

${ }^{1} \mathrm{H}$ NMR $\left(\delta[\mathrm{ppm}], \mathrm{C}_{6} \mathrm{D}_{6}, 20^{\circ} \mathrm{C}\right): 5.07 \quad(\mathrm{~s}, 2 \mathrm{H}$, COCHCO), 4.45 (sept, $\left.J=5.9 \mathrm{~Hz}, 2 \mathrm{H}, \mathrm{OCH}\left(\mathrm{CH}_{3}\right)_{2}\right), 4.16$ (sept, $\left.J=6.2 \mathrm{~Hz}, \quad 2 \mathrm{H}, \quad \mathrm{OCH}\left(\mathrm{CH}_{3}\right)_{2}\right), \quad 3.59 \quad(\mathrm{~s}, \quad 6 \mathrm{H}$, $\left.\mathrm{COOCH}_{3}\right), 1.66\left(\mathrm{~s}, 6 \mathrm{H}, \mathrm{CH}_{3} \mathrm{CO}\right) 1.49-1.30(\mathrm{~m}, 24 \mathrm{H}$, $\left.\mathrm{OCH}\left(\mathrm{CH}_{3}\right)_{2}\right) .{ }^{13} \mathrm{C} \quad\left\{{ }^{1} \mathrm{H}\right\} \quad$ NMR $\left(\delta\right.$ [ppm], d $\mathrm{d}_{8}$-toluene, $\left.20{ }^{\circ} \mathrm{C}\right): 186.8(\mathrm{CO}), 175.2(\mathrm{COO}), 85.5(\mathrm{COCHCO}), 66.0$ $\left(\mathrm{OCH}\left(\mathrm{CH}_{3}\right)_{2}\right), 63.1\left(\mathrm{OCH}\left(\mathrm{CH}_{3}\right)_{2}\right), 51.8\left(\mathrm{COOCH}_{3}\right), 28.0$ $\left(\mathrm{OCH}\left(\boldsymbol{C H}_{3}\right)_{2}\right), 25.5\left(\boldsymbol{C H}_{3} \mathrm{CO}\right), 25.3\left(\mathrm{OCH}\left(\boldsymbol{C H}_{3}\right)_{2}\right)$.

\section{$2.2\left[\mathrm{Al}\left(\mathrm{O}^{\mathrm{i}} \mathrm{Pr}\right)_{2}(\mathrm{etac})\right]_{2}$}

An amount of $0.999 \mathrm{~g}(4.89 \mathrm{mmol})$ of $\mathrm{Al}\left(\mathrm{O}^{\mathrm{i}} \mathrm{Pr}\right)_{3}$ was dissolved in $20 \mathrm{~mL}$ of toluene at room temperature followed by slow addition of $0.638 \mathrm{~g}(4.90 \mathrm{mmol})$ of ethyl acetoacetate under stirring. The clear reaction solution was stirred at room temperature for $12 \mathrm{~h}$ and then at $120{ }^{\circ} \mathrm{C}$ for additional $18 \mathrm{~h}$. The volatiles were removed in vacuo and a colorless oil was obtained. Yield $1.297 \mathrm{~g}(97 \%)$.

${ }^{1} \mathrm{H}$ NMR $\left(\delta[\mathrm{ppm}], \mathrm{C}_{6} \mathrm{D}_{6}, 20^{\circ} \mathrm{C}\right): 5.10 \quad(\mathrm{~s}, 2 \mathrm{H}$, $\mathrm{COCHCO}), \quad 4.60-4.00\left(\mathrm{~m}, 8 \mathrm{H}, \quad \mathrm{OCH}\left(\mathrm{CH}_{3}\right)_{2} / \mathrm{COOCH}_{2}\right.$ $\left.\mathrm{CH}_{3}\right), 1.68\left(\mathrm{~s}, 6 \mathrm{H}, \mathrm{CH}_{3} \mathrm{CO}\right), 1.48(\mathrm{~d}, J=6.2 \mathrm{~Hz}, 12 \mathrm{H}$, $\left.\mathrm{OCH}\left(\mathrm{CH}_{3}\right)_{2}\right), 1.39\left(\mathrm{~d}, J=5.9 \mathrm{~Hz}, 12 \mathrm{H}, \mathrm{OCH}\left(\mathrm{CH}_{3}\right)_{2}\right)$, 
$1.10\left(\mathrm{t}, J=7.1 \mathrm{~Hz}, 6 \mathrm{H}, \mathrm{COOCH}_{2} \mathrm{CH}_{3}\right) \cdot{ }^{13} \mathrm{C}\left\{{ }^{1} \mathrm{H}\right\} \mathrm{NMR}(\delta$ [ppm], $\mathrm{d}_{8}$-toluene, $\left.20{ }^{\circ} \mathrm{C}\right)$ : $186.5(\boldsymbol{C O}), 174.6(\boldsymbol{C O O}), 86.0$ (COCHCO), $65.9\left(\mathrm{OCH}\left(\mathrm{CH}_{3}\right)_{2}\right), 63.1\left(\mathrm{OCH}\left(\mathrm{CH}_{3}\right)_{2}\right), 61.1$ $\left(\mathrm{COOC} \mathrm{H}_{2} \mathrm{CH}_{3}\right), 28.0\left(\mathrm{OCH}\left(\boldsymbol{C H}_{3}\right)_{2}\right), 25.5\left(\boldsymbol{C H}_{3} \mathrm{CO}\right), 25.2$ $\left(\mathrm{OCH}\left(\mathrm{CH}_{3}\right)_{2}\right), 14.1\left(\mathrm{COOCH}_{2} \mathrm{CH}_{3}\right)$.

\section{$2.3\left[\mathrm{Al}\left(\mathrm{O}^{\mathrm{i}} \mathrm{Pr}\right)_{2}\left({ }^{\mathrm{i}} \mathrm{prac}\right)\right]_{2}$}

An amount of $1.006 \mathrm{~g}(4.93 \mathrm{mmol})$ of $\mathrm{Al}\left(\mathrm{O}^{\mathrm{i}} \mathrm{Pr}\right)_{3}$ was dissolved in $10 \mathrm{~mL}$ of toluene at room temperature followed by slow addition of $0.708 \mathrm{~g}(4.91 \mathrm{mmol})$ of iso-propyl acetoacetate under stirring. The clear reaction solution was stirred at $120{ }^{\circ} \mathrm{C}$ for $18 \mathrm{~h}$. The volatiles were removed in vасио and a colorless oil was obtained. Yield $1.388 \mathrm{~g}$ $(98 \%)$.

${ }^{1} \mathrm{H} \quad \mathrm{NMR} \quad\left(\delta \quad[\mathrm{ppm}], \mathrm{C}_{6} \mathrm{D}_{6}, 20^{\circ} \mathrm{C}\right): 5.39$ (quint, $\left.J=6.2 \mathrm{~Hz}, 2 \mathrm{H}, \mathrm{COOCH}\left(\mathrm{CH}_{3}\right)_{2}\right), 5.06(\mathrm{~s}, 2 \mathrm{H}, \mathrm{COCHCO})$, 4.50 (quint, $\left.J=5.8 \mathrm{~Hz}, 2 \mathrm{H}, \mathrm{OCH}\left(\mathrm{CH}_{3}\right)_{2}\right), 4.21$ (quint, $\left.J=6.2 \mathrm{~Hz}, 2 \mathrm{H}, \mathrm{OCH}\left(\mathrm{CH}_{3}\right)_{2}\right), 1.66\left(\mathrm{~s}, 6 \mathrm{H}, \mathrm{CH}_{3} \mathrm{CO}\right), 1.48$ $\left(\mathrm{d}, J=6.2 \mathrm{~Hz}, 12 \mathrm{H}, \mathrm{OCH}\left(\mathrm{CH}_{3}\right)_{2}\right), 1.39(\mathrm{~d}, J=5.8 \mathrm{~Hz}$, $\left.12 \mathrm{H}, \mathrm{OCH}\left(\mathrm{CH}_{3}\right)_{2}\right), 1.30(\mathrm{~d}, J=6.2 \mathrm{~Hz}, 6 \mathrm{H}, \mathrm{COOCH}$ $\left.\left(\mathrm{CH}_{3}\right)_{2}\right), 1.12\left(\mathrm{~d}, J=6.2 \mathrm{~Hz}, 6 \mathrm{H}, \mathrm{COOCH}\left(\mathrm{CH}_{3}\right)_{2}\right) .{ }^{13} \mathrm{C}$ $\left\{{ }^{1} \mathrm{H}\right\}$ NMR $\left(\delta\right.$ [ppm], d d $_{8}$-toluene, $\left.20{ }^{\circ} \mathrm{C}\right): 186.2(\boldsymbol{C O})$, $174.6(\mathrm{COO}), 86.5(\mathrm{COCHCO}), 68.6\left(\mathrm{COOCH}\left(\mathrm{CH}_{3}\right)_{2}\right)$, $65.8\left(\mathrm{OCH}\left(\mathrm{CH}_{3}\right)_{2}\right), \quad 63.2\left(\mathrm{OCH}\left(\mathrm{CH}_{3}\right)_{2}\right), \quad 28.0 \quad(\mathrm{OCH}$ $\left.\left(\boldsymbol{C H}_{3}\right)_{2}\right), \quad 25.5 \quad\left(\boldsymbol{C H}_{3} \mathrm{CO}\right), \quad 25.2 \quad\left(\mathrm{OCH}\left(\boldsymbol{C H}_{3}\right)_{2}\right), \quad 21.8$ $\left(\mathrm{COOCH}\left(\mathrm{CH}_{3}\right)_{2}\right)$.

\section{$2.4\left[\mathrm{Al}\left(\mathrm{O}^{\mathrm{i}} \mathrm{Pr}\right)_{2}\left({ }^{\mathrm{t}} \mathrm{buac}\right)\right]_{2}$}

An amount of $1.006 \mathrm{~g}(4.93 \mathrm{mmol})$ of $\mathrm{Al}\left(\mathrm{O}^{\mathrm{i}} \mathrm{Pr}\right)_{3}$ was dissolved in $10 \mathrm{~mL}$ of toluene at room temperature followed by slow addition of $0.709 \mathrm{~g}(4.92 \mathrm{mmol})$ tert-butyl acetoacetate under stirring. The clear reaction solution was stirred at $120{ }^{\circ} \mathrm{C}$ for $18 \mathrm{~h}$. The volatiles were then removed in vacuo and a white microcrystalline precipitate was obtained. Crystals suitable for single crystal XRD analysis were obtained upon crystallization from toluene at $4{ }^{\circ} \mathrm{C}$. Yield $1.331 \mathrm{~g}(89 \%)$

${ }^{1} \mathrm{H}$ NMR $\left(\delta[\mathrm{ppm}], \mathrm{C}_{6} \mathrm{D}_{6}, 20{ }^{\circ} \mathrm{C}\right): 5.06 / 5.05 / 4.98(\mathrm{~s}, 2 \mathrm{H}$, COCHCO $), 4.65-4.45\left(\mathrm{~m}, 2 \mathrm{H}, \mathrm{OCH}\left(\mathrm{CH}_{3}\right)_{2}\right), 4.35-4.15$ $\left(\mathrm{m}, 2 \mathrm{H}, \mathrm{OCH}\left(\mathrm{CH}_{3}\right)_{2}\right), 1.67$ (s, 6H, $\left.\mathrm{CH}_{3} \mathrm{CO}\right), 1.62-1.25$ $\left(\mathrm{m}, 42 \mathrm{H}, \mathrm{OCH}\left(\mathrm{CH}_{3}\right)_{2} / \mathrm{COOC}\left(\mathrm{CH}_{3}\right)_{3}\right) .{ }^{13} \mathrm{C}\left\{{ }^{1} \mathrm{H}\right\} \operatorname{NMR}(\delta$ [ppm], $\mathrm{d}_{8}$-toluene, $\left.20{ }^{\circ} \mathrm{C}\right):$ 189.9/185.3 (CO), $175.0(\boldsymbol{C O})$, 86.2/87.4 (COCHCO), 81.5/80.5/80.0 $\left(\mathrm{COOC}\left(\mathrm{CH}_{3}\right)_{3}\right), 65.8$ $\left(\mathrm{OCH}\left(\mathrm{CH}_{3}\right)_{2}\right), 63.1\left(\mathrm{OCH}\left(\mathrm{CH}_{3}\right)_{2}\right), 28.5\left(\mathrm{COOC}\left(\mathrm{CH}_{3}\right)_{3}\right)$, $28.0\left(\mathrm{OCH}\left(\boldsymbol{C H}_{3}\right)_{2}\right), 25.3\left(\boldsymbol{C H}_{3} \mathrm{CO}\right), 25.2\left(\mathrm{OCH}\left(\boldsymbol{C H}_{3}\right)_{2}\right)$.

\section{$2.5\left[\mathrm{Al}\left(\mathrm{O}^{\mathrm{i}} \mathrm{Pr}\right)_{2}(\mathrm{aaa})\right]_{2}$}

An amount of $0.998 \mathrm{~g}(4.89 \mathrm{mmol})$ of $\mathrm{Al}\left(\mathrm{O}^{\mathrm{i}} \mathrm{Pr}\right)_{3}$ was dissolved in $10 \mathrm{~mL}$ of toluene at room temperature followed by slow addition of $0.695 \mathrm{~g}(4.89 \mathrm{mmol})$ of allyl acetoacetate under stirring. The slightly turbid reaction solution was stirred at $120^{\circ} \mathrm{C}$ for $18 \mathrm{~h}$. The volatiles were removed in vacuo, the crude product washed with $5 \mathrm{~mL}$ of dichloromethane and a pale yellow, slightly turbid oil was obtained. Yield $1.300 \mathrm{~g}(93 \%)$.

${ }^{1} \mathrm{H}$ NMR $\left(\delta[\mathrm{ppm}], \mathrm{C}_{6} \mathrm{D}_{6}, 20{ }^{\circ} \mathrm{C}\right): 6.05-5.85(\mathrm{~m}, 2 \mathrm{H}$, $\mathrm{COOCH}_{2} \mathrm{CH}=\mathrm{CH}_{2}$ ), $5.10(\mathrm{~s}, 2 \mathrm{H}, \mathrm{COCHCO}), 5.30-5.00$ $\left(\mathrm{m}, 4 \mathrm{H}, \mathrm{COOCH}_{2} \mathrm{CH}=\mathrm{CH}_{2}\right), 4.95-4.55(\mathrm{~m}, 4 \mathrm{H}, \mathrm{COOC}$ $\boldsymbol{H}_{2} \mathrm{CH}=\mathrm{CH}_{2}$ ), 4.48 (quint, $J=5.9 \mathrm{~Hz}, 2 \mathrm{H}, \mathrm{OCH}\left(\mathrm{CH}_{3}\right)_{2}$ ), 4.18 (quint, $\left.J=6.2 \mathrm{~Hz}, 2 \mathrm{H}, \mathrm{OC} \boldsymbol{H}\left(\mathrm{CH}_{3}\right)_{2}\right), 1.66$ (s, $6 \mathrm{H}$, $\left.\mathrm{CH}_{3} \mathrm{CO}\right), 1.50-1.25\left(\mathrm{~m}, 24 \mathrm{H}, \mathrm{OCH}\left(\mathrm{CH}_{3}\right)_{2}\right) .{ }^{13} \mathrm{C}\left\{{ }^{1} \mathrm{H}\right\}$ NMR $\left(\delta[\mathrm{ppm}], \mathrm{d}_{8}\right.$-toluene, $\left.20{ }^{\circ} \mathrm{C}\right): 188.0(\boldsymbol{C O}), 174.0$ $(\mathrm{COO}), \quad 132.8 \quad\left(\mathrm{COOCH}_{2} \mathrm{CH}=\mathrm{CH}_{2}\right), \quad 117.5 \quad\left(\mathrm{COOCH}_{2}\right.$ $\left.\mathrm{CH}=\boldsymbol{C H}_{2}\right), \quad 86.0 \quad(\mathrm{COCHCO}), \quad 66.1 \quad\left(\mathrm{OCH}\left(\mathrm{CH}_{3}\right)_{2}\right), 66.0$ $\left(\mathrm{COOCH} \mathrm{H}_{2} \mathrm{CH}=\mathrm{CH}_{2}\right), \quad 63.1 \quad\left(\mathrm{OCH}\left(\mathrm{CH}_{3}\right)_{2}\right), \quad 28.0 \quad(\mathrm{OCH}$ $\left.\left(\boldsymbol{C H}_{3}\right)_{2}\right), 25.5\left(\boldsymbol{C H}_{3} \mathrm{CO}\right), 25.0\left(\mathrm{OCH}\left(\boldsymbol{C H}_{3}\right)_{2}\right)$.

\section{$2.6\left[\mathrm{Al}\left(\mathrm{O}^{\mathrm{i}} \mathrm{Pr}\right)_{2}(\text { meaa })\right]_{2}$}

A solution of $1.009 \mathrm{~g}(4.94 \mathrm{mmol})$ of $\mathrm{Al}\left(\mathrm{O}^{\mathrm{i}} \mathrm{Pr}\right)_{3}$ dissolved in $10 \mathrm{~mL}$ of toluene was stirred at $120^{\circ} \mathrm{C}$ for $18 \mathrm{~h}$. After cooling to room temperature $1.055 \mathrm{~g}(4.92 \mathrm{mmol})$ of 2-(methacryloyloxy)ethyl acetoacetate was slowly added under stirring. The clear reaction solution was stirred at room temperature for additional $18 \mathrm{~h}$. The volatiles were then removed in vасио and a colorless oil was obtained. Yield $1.696 \mathrm{~g}(96 \%)$.

${ }^{1} \mathrm{H} \quad \mathrm{NMR}\left(\delta[\mathrm{ppm}], \mathrm{C}_{6} \mathrm{D}_{6}, \quad 20{ }^{\circ} \mathrm{C}\right): 6.12(\mathrm{~s}, \quad 2 \mathrm{H}$, $\left.\mathrm{OC}(\mathrm{O}) \mathrm{C}\left(\mathrm{CH}_{3}\right)=\mathrm{CH}_{2}\right), 5.18\left(\mathrm{~s}, 2 \mathrm{H}, \mathrm{OC}(\mathrm{O}) \mathrm{C}\left(\mathrm{CH}_{3}\right)=\mathrm{CH}_{2}\right)$, $5.05(\mathrm{~s}, 2 \mathrm{H}, \mathrm{COCHCO}), 4.80-3.90\left(\mathrm{~m}, 12 \mathrm{H}, \mathrm{OCH}\left(\mathrm{CH}_{3}\right)_{2} /\right.$ $\left.\mathrm{OCH}_{2} \mathrm{CH}_{2} \mathrm{O}\right), 1.79\left(\mathrm{~s}, 6 \mathrm{H}, \mathrm{OC}(\mathrm{O}) \mathrm{C}\left(\mathrm{CH}_{3}\right)=\mathrm{CH}_{2}\right), 1.63(\mathrm{~s}$, $\left.6 \mathrm{H}, \mathrm{CH}_{3} \mathrm{CO}\right), 1.55-1.25\left(\mathrm{~m}, 24 \mathrm{H}, \mathrm{OCH}\left(\mathrm{CH}_{3}\right)_{2}\right) .{ }^{13} \mathrm{C}\left\{{ }^{1} \mathrm{H}\right\}$ NMR $\left(\delta[\mathrm{ppm}], \mathrm{d}_{8}\right.$-toluene, $\left.20^{\circ} \mathrm{C}\right): 187.5(\boldsymbol{C O}), 174.4$ $(\boldsymbol{C O O}), \quad 166.3\left(\mathrm{OC}(\mathrm{O}) \mathrm{C}\left(\mathrm{CH}_{3}\right)=\mathrm{CH}_{2}\right), 136.2 \quad(\mathrm{OC}(\mathrm{O}) \boldsymbol{C}$ $\left.\left(\mathrm{CH}_{3}\right)=\mathrm{CH}_{2}\right), 125.0\left(\mathrm{OC}(\mathrm{O}) \mathrm{C}\left(\mathrm{CH}_{3}\right)=\mathrm{CH}_{2}\right), 85.8(\mathrm{COCH}$ $\mathrm{CO}), 66.0\left(\mathrm{OCH}\left(\mathrm{CH}_{3}\right)_{2}\right), 63.1\left(\mathrm{OCH}\left(\mathrm{CH}_{3}\right)_{2}\right), 62.3\left(\mathrm{OCH}_{2}\right.$ $\left.\mathrm{CH}_{2} \mathrm{O}\right), 27.9\left(\mathrm{OCH}\left(\boldsymbol{C H}_{3}\right)_{2}\right), 25.4\left(\boldsymbol{C H}_{3} \mathrm{CO}\right), 25.0(\mathrm{OCH}$ $\left.\left(\boldsymbol{C H}_{3}\right)_{2}\right) 17.8\left(\mathrm{OC}(\mathrm{O}) \mathrm{C}\left(\boldsymbol{C} \mathrm{H}_{3}\right)=\mathrm{CH}_{2}\right)$.

\section{$2.7 \mathrm{Al}(\mathrm{meac})_{3}$}

An amount of $1.004 \mathrm{~g}(4.92 \mathrm{mmol})$ of $\mathrm{Al}\left(\mathrm{O}^{\mathrm{i}} \mathrm{Pr}\right)_{3}$ was dissolved in $10 \mathrm{~mL}$ of toluene at room temperature followed by slow addition of $1.710 \mathrm{~g}(14.71 \mathrm{mmol})$ of methyl acetoacetate under stirring. The clear reaction solution was then stirred at room temperature for $18 \mathrm{~h}$. The volatiles were removed in vacuo and the resulting colorless solid washed with $n$-pentane.

${ }^{1} \mathrm{H} \quad \mathrm{NMR} \quad\left(\delta \quad[\mathrm{ppm}], \mathrm{C}_{6} \mathrm{D}_{6}, \quad 20{ }^{\circ} \mathrm{C}\right): 5.17 \quad(\mathrm{~s}, \quad 3 \mathrm{H}$, COCHCO), 3.43/3.38/3.34/3.33 (s, 9H, OCH $H_{3}$ ), 1.83/1.82/ 1.80/1.79/1.77 (s, 9H, CH $\left.\boldsymbol{H}_{3} \mathrm{CO}\right) .{ }^{13} \mathrm{C}\left\{{ }^{1} \mathrm{H}\right\}$ NMR $(\delta[\mathrm{ppm}]$, $\mathrm{d}_{8}$-toluene, $20{ }^{\circ} \mathrm{C}$ ): 188.3/188.2/187.9/187.8 (CO), 174.8/ 
174.6 (COO), 85.0/84.7/84.5 (COCHCO), 51.0/50.9/50.8/ $50.7\left(\mathrm{OCH}_{3}\right), 25.8\left(\mathrm{CH}_{3} \mathrm{CO}\right)$.

\section{$2.8 \mathrm{Al}(\text { etac })_{3}$}

An amount of $1.001 \mathrm{~g}(4.90 \mathrm{mmol})$ of $\mathrm{Al}\left(\mathrm{O}^{\mathrm{i}} \mathrm{Pr}\right)_{3}$ was dissolved in $20 \mathrm{~mL}$ of toluene at room temperature and $1.914 \mathrm{~g}(14.71 \mathrm{mmol})$ of ethyl acetoacetate was slowly added under stirring. The clear reaction solution was stirred at room temperature for $18 \mathrm{~h}$. The volatiles were removed in vacuo and a white solid was obtained.

${ }^{1} \mathrm{H}$ NMR $\left(\delta\right.$ [ppm], $\left.\mathrm{C}_{6} \mathrm{D}_{6}, 20{ }^{\circ} \mathrm{C}\right): 5.18 / 5.17$ (s, 3H, COCHCO), 4.15-3.85 (m, 6H, OCH $\mathrm{CH}_{3}$ ), 1.85/1.84/1.81/ 1.80 (s, 9H, $\left.\mathrm{CH}_{3} \mathrm{CO}\right), 1.00 / 0.99 / 0.91 / 0.90(\mathrm{t}, J=7.1 \mathrm{~Hz}$, $\left.9 \mathrm{H}, \mathrm{OCH}_{2} \mathrm{CH}_{3}\right) .{ }^{13} \mathrm{C}\left\{{ }^{1} \mathrm{H}\right\}$ NMR $\left(\delta[\mathrm{ppm}], \mathrm{d}_{8}\right.$-toluene, $\left.20{ }^{\circ} \mathrm{C}\right): 188.1 / 188.0 / 187.7 / 187.6(C O), 174.5 / 174.3 / 174.2$ (COO), 85.2/85.0/84.8 (COCHCO), 60.4/60.3/60.2/60.1 $\left(\mathrm{OCH}_{2} \mathrm{CH}_{3}\right), 25.9\left(\boldsymbol{C H}_{3} \mathrm{CO}\right), 14.1 / 13.9\left(\mathrm{OCH}_{2} \boldsymbol{C H}_{3}\right) .{ }^{27} \mathrm{Al}$ NMR $\left(\delta[\mathrm{ppm}], \mathrm{d}_{8}\right.$-toluene, $\left.20{ }^{\circ} \mathrm{C}\right): 4.78 \mathrm{ppm}$.

\section{$2.9 \mathrm{Al}\left({ }^{\mathrm{i}} \text { prac }\right)_{3}$}

An amount of $1.005 \mathrm{~g}(4.92 \mathrm{mmol})$ of $\mathrm{Al}\left(\mathrm{O}^{\mathrm{i}} \mathrm{Pr}\right)_{3}$ was dissolved in $10 \mathrm{~mL}$ of toluene at room temperature and $2.125 \mathrm{~g}$ (14.74 mmol) of iso-propyl acetoacetate was slowly added under stirring. The clear reaction solution was stirred at room temperature for $18 \mathrm{~h}$. The volatiles were removed in vacuo and a white, partially crystalline solid was obtained.

${ }^{1} \mathrm{H} \quad \mathrm{NMR} \quad\left(\delta[\mathrm{ppm}], \mathrm{C}_{6} \mathrm{D}_{6}, 20^{\circ} \mathrm{C}\right): 5.16 \quad(\mathrm{~s}, 3 \mathrm{H}$, $\mathrm{COCHCO}), 5.12-4.94\left(\mathrm{~m}, 3 \mathrm{H}, \mathrm{OCH}\left(\mathrm{CH}_{3}\right)_{2}\right), 1.86 / 1.84 /$ $1.81 / 1.79$ (s, 9H, $\left.\mathrm{CH}_{3} \mathrm{CO}\right), 1.20-0.95(\mathrm{~m}, 18 \mathrm{H}, \mathrm{OCH}$ $\left.\left(\mathrm{CH}_{3}\right)_{2}\right) .{ }^{13} \mathrm{C}\left\{{ }^{1} \mathrm{H}\right\}$ NMR $\left(\delta[\mathrm{ppm}], \mathrm{d}_{8}\right.$-toluene, $\left.20{ }^{\circ} \mathrm{C}\right)$ : 187.9/187.6/187.4/187.3 (CO), 175.1/175.0/173.9/173.8 (COO), 85.6/85.4/85.1 (COCHCO), 67.9/67.8/67.7/67.6 $\left(\mathrm{OCH}\left(\mathrm{CH}_{3}\right)_{2}\right), \quad 25.8 / 25.7 \quad\left(\mathrm{CH}_{3} \mathrm{CO}\right), \quad 21.7 / 21.6 / 21.5 / 21.4$ $\left(\mathrm{OCH}\left(\mathrm{CH}_{3}\right)_{2}\right)$.

\section{$2.10 \mathrm{Al}\left({ }^{\mathrm{t}} \mathrm{buac}\right)_{3}$}

An amount of $0.999 \mathrm{~g}(4.89 \mathrm{mmol})$ of $\mathrm{Al}\left(\mathrm{O}^{\mathrm{i}} \mathrm{Pr}\right)_{3}$ was dissolved in $10 \mathrm{~mL}$ of toluene at room temperature and $2.318 \mathrm{~g}(14.65 \mathrm{mmol})$ tert-butyl acetoacetate was slowly added under stirring. The clear reaction solution was stirred at room temperature for $18 \mathrm{~h}$. The volatiles were then removed in vacuo and the resulting white, partially crystalline solid washed with dichloromethane. Yield $2.278 \mathrm{~g}$ (93\%).

${ }^{1} \mathrm{H}$ NMR $\left(\delta[\mathrm{ppm}], \mathrm{C}_{6} \mathrm{D}_{6}, 20{ }^{\circ} \mathrm{C}\right): 5.13 / 5.10 / 5.08$ (s, 3H, COCHCO), 1.83/1.82/1.79 (s, 9H, $\mathrm{CH}_{3} \mathrm{CO}$ ), 1.46/1.45 (s, $\left.27 \mathrm{H}, \mathrm{OC}\left(\mathrm{CH}_{3}\right)_{3}\right) \cdot{ }^{13} \mathrm{C}\left\{{ }^{1} \mathrm{H}\right\}$ NMR $\left(\delta[\mathrm{ppm}], \mathrm{d}_{8}\right.$-toluene, $\left.20{ }^{\circ} \mathrm{C}\right): 187.2 / 187.0 / 186.9 / 186.6($ CO $), 174.5 / 174.4 / 174.3 /$ 174.1 (COO), 86.4/86.2/86.1/86.8 (COCHCO), 80.6/80.3/
80.2/80.0 $\left(\mathrm{OC}\left(\mathrm{CH}_{3}\right)_{3}\right), 28.8 / 28.3\left(\mathrm{OC}\left(\boldsymbol{C H}_{3}\right)_{3}\right), 26.0 / 25.9 /$ $25.7\left(\mathrm{CH}_{3} \mathrm{CO}\right)$.

$2.11 \mathrm{Al}(\text { aaa })_{3}$

An amount of $0.999 \mathrm{~g}(4.89 \mathrm{mmol})$ of $\mathrm{Al}\left(\mathrm{O}^{\mathrm{i}} \mathrm{Pr}\right)_{3}$ was dissolved in $10 \mathrm{~mL}$ of toluene at room temperature and $2.084 \mathrm{~g}$ (14.66 mmol) of allyl acetoacetate was slowly added under stirring. The slightly turbid reaction solution was stirred at room temperature for $18 \mathrm{~h}$. The volatiles were then removed in vacuo and the resulting orange oil washed with dichloromethane. Yield $2.167 \mathrm{~g}(98 \%)$.

${ }^{1} \mathrm{H}$ NMR $\left(\delta[\mathrm{ppm}], \mathrm{C}_{6} \mathrm{D}_{6}, 20{ }^{\circ} \mathrm{C}\right): 5.95-5.55(\mathrm{~m}, 3 \mathrm{H}$, $\left.\mathrm{OCH}_{2} \mathrm{CH}=\mathrm{CH}_{2}\right), 5.15$ (s, 3H, COCHCO), 5.10-4.85 (m, $\left.6 \mathrm{H}, \mathrm{OCH}_{2} \mathrm{CH}=\mathrm{CH}_{2}\right), 4.60-4.30\left(\mathrm{~m}, 6 \mathrm{H}, \mathrm{OCH}_{2} \mathrm{CH}=\mathrm{CH}_{2}\right)$, $1.81 / 1.79 / 1.78 / 1.76$ (s, 9H, $\left.\mathrm{CH}_{3} \mathrm{CO}\right) .{ }^{13} \mathrm{C}\left\{{ }^{1} \mathrm{H}\right\}$ NMR $(\delta$ [ppm], $\mathrm{d}_{8}$-toluene, $\left.20{ }^{\circ} \mathrm{C}\right): 188.6 / 188.4 / 188.2 / 188.0(C O)$, 174.1/174.0/173.9/173.8 (COO), 132.7/132.6/132.5/132.4 $\left(\mathrm{OCH}_{2} \mathrm{CH}=\mathrm{CH}_{2}\right), \quad 117.8 / 117.6 / 117.4 / 117.3 \quad\left(\mathrm{OCH}_{2} \mathrm{CH}=\right.$ $\mathrm{CH}_{2}$ ), 85.1/84.9/84.8/84.7 (COCHCO), 65.2/65.0/64.8 $\left(\mathrm{OCH}_{2} \mathrm{CH}=\mathrm{CH}_{2}\right)$, 25.9/25.8 $\left(\mathrm{CH}_{3} \mathrm{CO}\right)$.

\section{$2.12 \mathrm{Al}(\text { meaa })_{3}$}

An amount of $1.001 \mathrm{~g}(4.90 \mathrm{mmol}) \mathrm{Al}\left(\mathrm{O}^{\mathrm{i}} \mathrm{Pr}\right)_{3}$ was dissolved in $10 \mathrm{~mL}$ of toluene at room temperature and $3.153 \mathrm{~g}(14.72 \mathrm{mmol})$ of 2-(methacryloyloxy)ethyl acetoacetate was slowly added under stirring. The clear reaction solution was stirred at room temperature for $18 \mathrm{~h}$. The volatiles were removed in vacuo, the crude product washed with dichloromethane and a colorless rubber-like mass was obtained. Yield $3.114 \mathrm{~g}(95 \%)$.

${ }^{1} \mathrm{H}$ NMR $\left(\delta\right.$ [ppm], $\left.\mathrm{C}_{6} \mathrm{D}_{6}, 20{ }^{\circ} \mathrm{C}\right): 6.12 / 6.08$ (s, 3H, OC $\left.(\mathrm{O}) \mathrm{C}\left(\mathrm{CH}_{3}\right)=\mathrm{CH}_{2}\right), \quad 5.25-5.15 \quad\left(\mathrm{~m}, \quad 3 \mathrm{H}, \quad \mathrm{OC}(\mathrm{O}) \mathrm{C}\left(\mathrm{CH}_{3}\right)=\right.$ $\left.\mathrm{CH}_{2}\right), 5.14(\mathrm{~s}, 3 \mathrm{H}, \mathrm{COCHCO}), 4.40-3.90(\mathrm{~m}, 12 \mathrm{H}$, $\left.\mathrm{OCH}_{2} \mathrm{CH}_{2} \mathrm{O}\right), 1.85-1.75\left(\mathrm{~m}, 18 \mathrm{H}, \mathrm{OC}(\mathrm{O}) \mathrm{C}\left(\mathrm{CH}_{3}\right)=\mathrm{CH}_{2}\right)$, 1.85 (s, 9H, CH $\left.\boldsymbol{H}_{3} \mathrm{CO}\right) .{ }^{13} \mathrm{C}\left\{{ }^{1} \mathrm{H}\right\}$ NMR $\left(\delta[\mathrm{ppm}], \mathrm{d}_{8}\right.$-toluene, $\left.20{ }^{\circ} \mathrm{C}\right): 189.2 / 189.0 / 188.6 / 188.5(\boldsymbol{C O}), 174.2 / 174(\boldsymbol{C O O})$, $166.3 / 166.2 / 166.1 \quad\left(\mathrm{OC}(\mathrm{O}) \mathrm{C}\left(\mathrm{CH}_{3}\right)=\mathrm{CH}_{2}\right), 136.1 \quad(\mathrm{OC}(\mathrm{O})$ $\left.\boldsymbol{C}\left(\mathrm{CH}_{3}\right)=\mathrm{CH}_{2}\right), \quad 125.3 / 125.0 / 124.8 \quad\left(\mathrm{OC}(\mathrm{O}) \mathrm{C}\left(\mathrm{CH}_{3}\right)=C \mathrm{H}_{2}\right)$, 85.1/84.9/84.8 (COCHCO), 62.5/62.4/62.3/62.2 $\left(\mathrm{OCH}_{2}\right.$ $\left.\boldsymbol{C} \mathrm{H}_{2} \mathrm{O}\right), 25.9 / 15.1\left(\boldsymbol{C} \mathrm{H}_{3} \mathrm{CO}\right), 17.9\left(\mathrm{OC}(\mathrm{O}) \mathrm{C}\left(\boldsymbol{C} \mathrm{H}_{3}\right)=\mathrm{CH}_{2}\right)$.

\section{Results and discussion}

First attempts to prepare compounds $\left[\mathrm{Al}\left(\mathrm{O}^{\mathrm{i}} \mathrm{Pr}\right)_{2}(\beta\right.$-ketoesterate $)]_{n}$ by reaction of $\left[\mathrm{Al}\left(\mathrm{O}^{\mathrm{i}} \mathrm{Pr}\right)_{3}\right]_{4}$ with one molar equivalent of $\beta$-ketoester per $\mathrm{Al}$ at room temperature according to literature procedures for the modification of aluminum alkoxides with acetylacetone [5] and of titanium alkoxides with $\beta$-ketoesters $[8,9]$ did not yield the desired products. Even after prolonged reaction times at room 
temperature, only a mixture of $\mathrm{Al}(\beta \text {-ketoesterate })_{3}$ and unsubstituted $\left[\mathrm{Al}\left(\mathrm{O}^{\mathrm{i}} \mathrm{Pr}\right)_{3}\right]_{4}$ was obtained for all esters used (Eq. 1).

$$
\begin{aligned}
& 3\left[\mathrm{Al}\left(\mathrm{O}^{\mathrm{i}} \mathrm{Pr}\right)_{3}\right]_{4}+12 \beta \text {-ketoester } \underset{\text { Toluene }}{\stackrel{\text { r.t., } 18 \mathrm{~h}}{\longrightarrow}} 2\left[\mathrm{Al}\left(\mathrm{O}^{\mathrm{i}} \mathrm{Pr}\right)_{3}\right]_{4} \\
& \quad+4 \mathrm{Al}(\beta \text {-ketoesterate })_{3}+12^{\mathrm{i}} \mathrm{Pr} \mathrm{OH}
\end{aligned}
$$

Coordination of the $\beta$-ketoesterate ligand during this reaction was monitored by ${ }^{1} \mathrm{H}$ NMR spectroscopy, showing the disappearance of the signals of the $\mathrm{CH}_{2}$ group between the two carbonyl groups of the non-coordinated esters (2.92-3.02 ppm) and the appearance of the signals for the corresponding $\mathrm{CH}$ proton of the deprotonated and coordinated ligand (5.18-5.08 ppm).

The exclusive formation of $\mathrm{Al}(\beta \text {-ketoesterate })_{3}$ indicates that intermediate $\left[\mathrm{Al}\left(\mathrm{O}^{\mathrm{i}} \mathrm{Pr}\right)_{x}(\beta \text {-ketoesterate })_{3-x}\right]_{n}$ species react more rapidly with $\beta$-ketoesters than $\left[\mathrm{Al}\left(\mathrm{O}^{\mathrm{i}} \mathrm{Pr}\right)_{3}\right]_{4}$.

Heating the reaction solution, containing $\mathrm{Al}(\beta$-ketoesterate $)_{3}$ and $\left[\mathrm{Al}\left(\mathrm{O}^{\mathrm{i}} \mathrm{Pr}\right)_{3}\right]_{4}$ along with liberated iso-propanol, to $120{ }^{\circ} \mathrm{C}$ overnight resulted in the formation of the anticipated $\left[\mathrm{Al}\left(\mathrm{O}^{\mathrm{i}} \mathrm{Pr}\right)_{2}(\beta \text {-ketoesterate })\right]_{n}$ derivatives (Eq. 2). Reaction of isolated $\mathrm{Al}(\beta \text {-ketoesterate })_{3}$ and $\left[\mathrm{Al}\left(\mathrm{O}^{\mathrm{i}} \mathrm{Pr}\right)_{3}\right]_{4}$ in appropriate stoichiometric ratios in toluene (without free alcohol) gave the same results. After cooling to room temperature no redistribution to $\mathrm{Al}(\beta \text {-ketoesterate })_{3}$ and $\left[\mathrm{Al}\left(\mathrm{O}^{\mathrm{i}} \mathrm{Pr}\right)_{3}\right]_{4}$ occurred. This proves, that the monosubstituted dimer is the most stable species for this $\mathrm{Al} / \beta$-ketoester ratio and the formation of $\mathrm{Al}(\beta \text {-ketoesterate })_{3}$ at room temperature is due to a kinetic effect.

$$
\begin{aligned}
& {\left[\mathrm{Al}\left(\mathrm{O}^{\mathrm{i}} \mathrm{Pr}\right)_{3}\right]_{4}+2 \mathrm{Al}(\beta \text {-ketoesterate })_{3} \underset{\text { Toluene }}{\stackrel{120^{\circ} \mathrm{C}, 18 \mathrm{~h}}{\longrightarrow}}} \\
& 3\left[\mathrm{Al}\left(\mathrm{O}^{\mathrm{i}} \mathrm{Pr}\right)_{2}(\beta \text {-ketoesterate })\right]_{2}
\end{aligned}
$$

The same result was also obtained for an alternative preparation route, where a toluene solution of $\left[\mathrm{Al}\left(\mathrm{O}^{\mathrm{i}} \mathrm{Pr}\right)_{3}\right]_{4}$ was thermally pre-treated. This is known to cause de-oligomerization of the tetrameric units $[2,10,11]$. According to literature, the solution consists mainly of dimeric and trimeric species after fast cooling to room temperature, but some tetrameric $\left[\mathrm{Al}\left(\mathrm{O}^{\mathrm{i}} \mathrm{Pr}\right)_{3}\right]_{4}$ is still present or reformed. This was confirmed by ${ }^{27} \mathrm{Al} \mathrm{NMR}$ spectroscopy, showing a broad signal for pentacoordinate $\mathrm{Al}$ of trimeric species in the range from about 45 to $15 \mathrm{ppm}$

Fig. 2 Tetra-, tri- and dimeric form of $\left[\mathrm{Al}(\mathrm{OR})_{3}\right]_{n}(n=2-4)$
[12], as well as a sharp signal at about 0 ppm for hexacoordinate $\mathrm{Al}$ of the tetramer, besides signals from about 80 to $50 \mathrm{ppm}$ for tetracoordinate $\mathrm{Al}$ of di-, tri-, and tetrameric species (Fig. 2). ${ }^{1} \mathrm{H}$ NMR and EXSY spectroscopy additionally shows fast exchange between the alkoxo groups of all species present in the solution. After de-oligomerization of tetrameric $\left[\mathrm{Al}\left(\mathrm{O}^{\mathrm{i}} \mathrm{Pr}\right)_{3}\right]_{4}$, the solution was cooled to room temperature, the $\beta$-ketoester was added and allowed to react for several hours. The resulting product was identified as $\left[\mathrm{Al}\left(\mathrm{O}^{\mathrm{i}} \mathrm{Pr}\right)_{2}\right.$ $(\beta$-ketoesterate $)]_{2}$, which means that pre-heating of the parent alkoxide and subsequent addition of ligand results in the same products as the in-situ heating of a mixture of the metal alkoxide and the ligand. This result shows (i) that reformation of $\left[\mathrm{Al}\left(\mathrm{O}^{\mathrm{i}} \mathrm{Pr}\right)_{3}\right]_{4}$ from the dimer and trimer is slow at room temperature and (ii) that the dimer and trimer react faster with the $\beta$-ketoesters than the tetramer, and also faster than intermediate $\left[\mathrm{Al}\left(\mathrm{O}^{\mathrm{i}} \mathrm{Pr}\right)_{x}(\beta \text {-ketoesterate })_{3-x}\right]_{n}$, giving directly the monosubstituted compounds.

The alternative synthetic pathway, i.e. thermal pretreatment of the alkoxide, also opens the possibility to use temperature-sensitive ligands for modification. For example, gelation occurs when 2-(methacryloyloxy)ethyl acetoacetate (meaa-H), with its polymerizable methacrylic double bond, is reacted with $\mathrm{Al}\left(\mathrm{O}^{\mathrm{i}} \mathrm{Pr}\right)_{3}$ at elevated temperatures, and no defined product could be isolated. In contrast, thermal de-oligomerization of the metal alkoxide tetramer and coordination of the ester under mild conditions (room temperature) yielded a well defined product, useable as precursor for inorganic-organic hybrid materials. Characterization by NMR spectroscopy showed the expected coordination of the $\beta$-ketoester giving rise to a signal at $5.05 \mathrm{ppm}$ for the $\mathrm{COCHCO}$ proton. Signals for the $=\mathrm{CH}_{2}$ protons of the methacrylate at 6.12 and $5.18 \mathrm{ppm}$ showed preservation of the polymerizable double bond (Fig. 3). The small signal at 6.06 ppm results from minor impurities of $\mathrm{Al}(\text { meaa })_{3}$, which is formed because in the pre-treated $\mathrm{Al}\left(\mathrm{O}^{\mathrm{i}} \mathrm{Pr}\right)_{3}$ solution still some tetrameric $\left[\mathrm{Al}\left(\mathrm{O}^{\mathrm{i}} \mathrm{Pr}\right)_{3}\right]_{4}$ is present, as mentioned above. Additional signals at about $7.10-7.00 \mathrm{ppm}$ and at $2.10 \mathrm{ppm}$ result from residual toluene.

The allyl acetoacetate derivative as an alternative compound bearing a polymerizable group can be

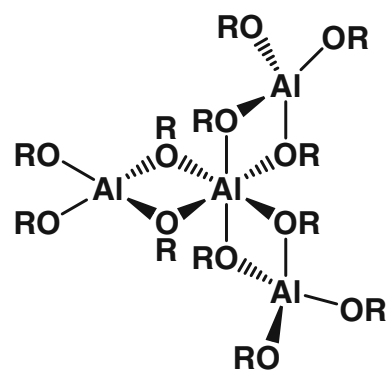

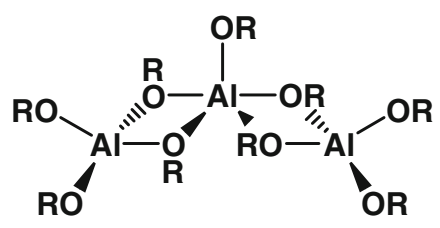

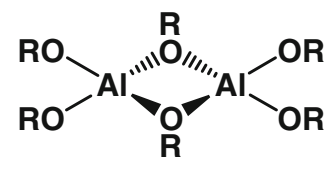




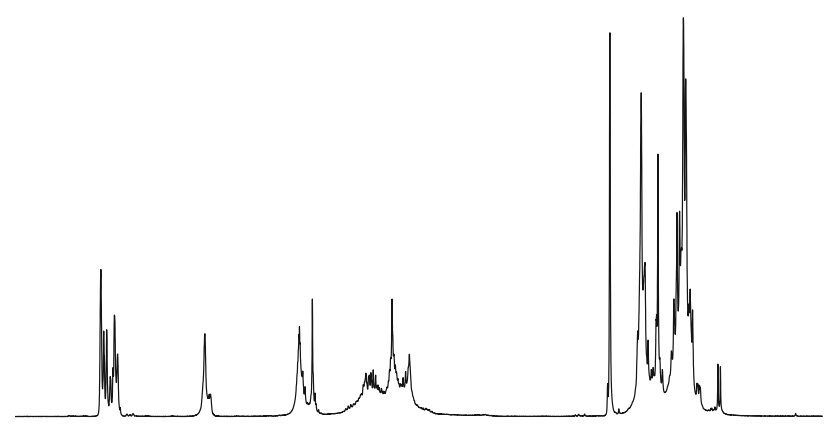

$\begin{array}{lllllllllllllll}7.5 & 7.0 & 6.5 & 6.0 & 5.5 & 5.0 & 4.5 & 4.0 & 3.5 & 3.0 & 2.5 & 2.0 & 1.5 & 1.0 & \mathrm{ppm}\end{array}$

Fig. $3{ }^{1} \mathrm{H}$ NMR spectrum of $\left[\mathrm{Al}\left(\mathrm{O}^{\mathrm{i}} \mathrm{Pr}\right)_{2}(\text { meaa })\right]_{2}$ in $\mathrm{C}_{6} \mathrm{D}_{6}$

synthesized by the "conventional" route because the ligand has a sufficient thermal stability.

All $\mathrm{Al}(\mathrm{OR})_{2}\left(\beta\right.$-ketoesterate) derivatives show two ${ }^{1} \mathrm{H}$ NMR signals for the methine protons of the iso-propoxo groups, indicating the existence of two chemically different ligands. According to the X-ray analysis reported below, the derivatives are asymmetrically substituted, alkoxobridged dimers (Fig. 4). The structure type is the same as that of $\left[\mathrm{Al}\left(\mathrm{OSiMe}_{3}\right)_{2}(\mathrm{acac})\right]_{2}$ [5]. In this structure, one aluminum center is tetrahedrally coordinated by two bridging and two terminal alkoxo groups, whereas the other aluminum center is octahedrally coordinated by two chelating $\beta$-ketoesterate ligands and the two bridging alkoxo groups. Given the asymmetric nature of the $\beta$-ketoesterate ligands, this results in three possible isomers, one $\mathrm{C}_{1}$ and two $\mathrm{C}_{2}$ symmetric (Fig. 4), each forming a pair of enantiomers, giving 6 stereoisomers overall. In the NMR spectrum, the $\mathrm{C}_{2}$ symmetric complexes should show one set of signals for the ester ligands and two sets for the isopropoxo groups (one for the bridging and one for the terminal) and splitting of these signals in two sets each for the $\mathrm{C}_{1}$ symmetric complex. Since, in some cases, more than two signals were observed for the $\beta$-ketoesterate ligands and only two signals for the methine protons of the isopropoxo groups, different isomers coexist in solution for which the iso-propoxo signals are not distinguishable. All signals were assigned by COSY, HSQC and HMBC NMR

Fig. 4 Possibly isomers of $\left[\mathrm{Al}(\mathrm{OR})_{2}(\beta \text {-ketoeterate })\right]_{2}$ (only one enantiomer shown for each geometry) spectroscopy, showing the coexistence of more than one species in solution, but with one clearly dominating form. The methine signals of the iso-propoxo are clearly assigned to bridging and terminal groups, but in the $\mathrm{CH}_{3}$ region multi signal overlap was observed and thus a definite assignment is difficult. The $\left[\mathrm{Al}\left(\mathrm{O}^{\mathrm{i}} \mathrm{Pr}\right)_{2}(\beta \text {-ketoesterate })\right]_{2}$ complexes also show small signals beside a sharp main signal for the $\mathrm{COCHCO}$ proton. Only $\left[\mathrm{Al}\left(\mathrm{O}^{\mathrm{i}} \mathrm{Pr}\right)_{2}\left({ }^{\mathrm{t}} \text { buac }\right)\right]_{2}$ gave three signals of almost equal intensity.

Intermolecular ligand exchange between the different species was observed in EXSY experiments for all complexes. For the complexes $\left[\mathrm{Al}\left(\mathrm{O}^{\mathrm{i} P r}\right)_{2}(\text { meac })\right]_{2},\left[\mathrm{Al}\left(\mathrm{O}^{\mathrm{i}} \mathrm{Pr}\right)_{2}\right.$ ( ${ }^{\mathrm{i}}$ prac $\left.)\right]_{2}$, and $\left[\mathrm{Al}\left(\mathrm{O}^{\mathrm{i}} \mathrm{Pr}\right)_{2}\left({ }^{\mathrm{t}} \text { buac }\right)\right]_{2}$ splitting of the $\mathrm{CH}_{3}$ protons of the methoxo, iso-propoxo and tert-butoxo ester groups, respectively, was also observed in the ${ }^{1} \mathrm{H}$ NMR spectra. Whereas this results in two signals of equal intensity for the ${ }^{i}$ prac and ${ }^{t}$ buac derivative, one main signal at $3.59 \mathrm{ppm}$ was found for the meac derivative, along with three smaller signals at $3.71,3.52$, and 3.24 ppm. In Fig. 5

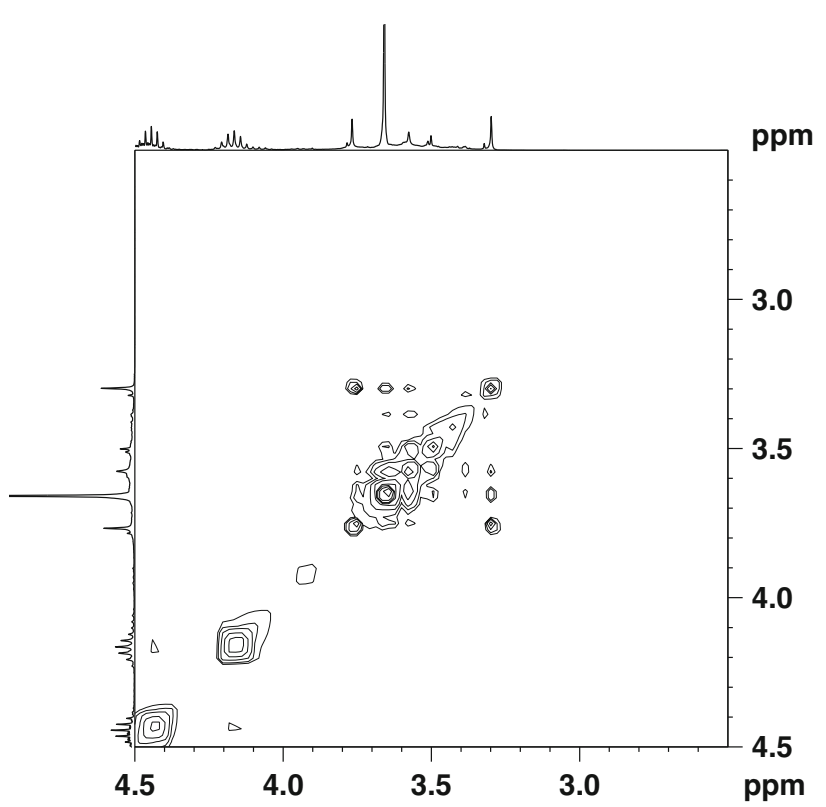

Fig. 5 EXSY spectrum of $\left[\mathrm{Al}\left(\mathrm{O}^{\mathrm{i}} \mathrm{Pr}\right)_{2}(\text { meac })\right]_{2}$ in $\mathrm{d}_{8}$-toluene, $\mathrm{OCH}_{3^{-}}$ region
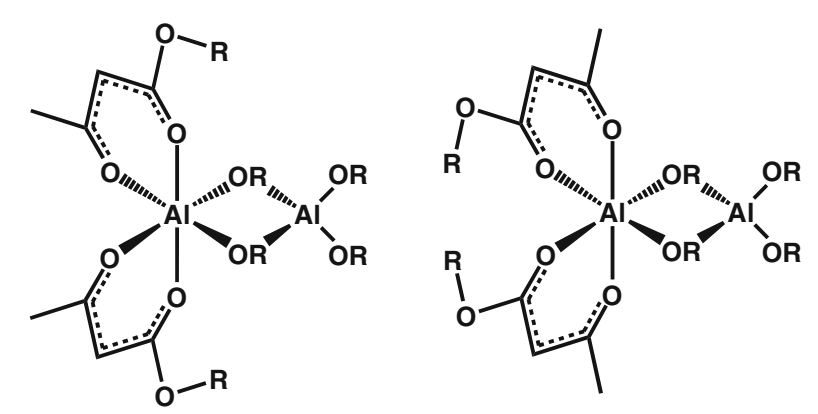

$\mathrm{C}_{1}$

$\mathrm{C}_{2}$ 
Table $1{ }^{1} \mathrm{H}$ NMR shifts of iso-propoxo groups of $\left[\mathrm{Al}\left(\mathrm{O}^{\mathrm{i}} \mathrm{Pr}\right)_{2}(\beta \text {-ketoesterate })\right]_{2}\left(\beta\right.$-ketoesterate $=$ meac, etac, ${ }^{\mathrm{i}}$ prac, ${ }^{\mathrm{t}}$ buac $)$ compared to

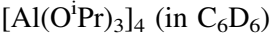

\begin{tabular}{|c|c|c|c|c|}
\hline & $\mathrm{OC} \boldsymbol{H}\left(\mathrm{CH}_{3}\right)_{2}$ (bridging) & $\mathrm{OC} \boldsymbol{H}\left(\mathrm{CH}_{3}\right)_{2}$ (terminal) & $\mathrm{OCH}\left(\mathrm{CH}_{3}\right)_{2}$ (bridging) & $\overline{\mathrm{OCH}\left(\mathrm{CH}_{3}\right)_{2} \text { (terminal) }}$ \\
\hline$\left[\mathrm{Al}\left(\mathrm{O}^{\mathrm{i}} \mathrm{Pr}\right)_{3}\right]_{4}$ & 4.69 & 4.41 & $1.68 / 1.39^{\mathrm{a}}$ & 1.32 \\
\hline$\left[\mathrm{Al}\left(\mathrm{O}^{\mathrm{i}} \mathrm{Pr}\right)_{2}(\mathrm{meac})\right]_{2}$ & 4.45 & 4.16 & $1.49-1.30^{\mathrm{b}}$ & $1.49-1.30^{\mathrm{b}}$ \\
\hline$\left[\mathrm{Al}\left(\mathrm{O}^{\mathrm{i}} \mathrm{Pr}\right)_{2}(\mathrm{etac})\right]_{2}$ & 4.48 & 4.18 & 1.48 & 1.39 \\
\hline$\left[\mathrm{Al}\left(\mathrm{O}^{\mathrm{i}} \mathrm{Pr}\right)_{2}\left({ }^{\mathrm{i}} \mathrm{prac}\right)\right]_{2}$ & 4.50 & 4.21 & 1.48 & 1.39 \\
\hline$\left[\mathrm{Al}\left(\mathrm{O}^{\mathrm{i}} \mathrm{Pr}\right)_{2}\left({ }^{\mathrm{t}} \mathrm{buac}\right)\right]_{2}$ & 4.55 & 4.28 & $1.62-1.25^{\mathrm{c}}$ & $1.62-1.25^{\mathrm{c}}$ \\
\hline
\end{tabular}

${ }^{a}$ Splitting of bridging iso-propoxo groups [2]

b Overlap of briging and terminal $\mathrm{OCH}\left(\mathrm{CH}_{3}\right)_{2}$

c Overlap with $\mathrm{OC}\left(\mathrm{CH}_{3}\right)_{3}$

the corresponding region of the EXSY spectrum is reproduced, showing exchange between all of these four signals.

In accordance with the NMR spectra of $\left[\mathrm{Al}\left(\mathrm{O}^{\mathrm{i}} \mathrm{Pr}\right)_{3}\right]_{4}$ (see below), the signals for the bridging groups are shifted to lower field. The shifts of the $\mathrm{O}^{\mathrm{i}} \mathrm{Pr}$ methine protons for the non-functional $\mathrm{Al}(\mathrm{OR})_{2}(\beta$-ketoesterate $)$ derivatives are compared with that of $\left[\mathrm{Al}\left(\mathrm{O}^{\mathrm{i}} \mathrm{Pr}\right)_{3}\right]_{4}$ in Table 1 . The methine proton signals for all complexes are upfield shifted, corresponding to the reduced Lewis acidity of the substituted aluminum center. A slight trend to higher ppm values with increasing size of the ester OR groups for the ${ }^{1} \mathrm{H}$ NMR signals of the methine protons is observed. Corresponding ${ }^{13} \mathrm{C}$ NMR signals are not influenced by the ester OR group.
Crystals were obtained for the complex $\left[\mathrm{Al}\left(\mathrm{O}^{\mathrm{i}} \mathrm{Pr}\right)_{2}\right.$ ('buac) $]_{2}$, and an X-ray crystal structure analysis was carried out. Although the quality of the data set was affected by formation of thin platelets and partial decomposition of the crystal during the measurement, the dimeric nature of the compound with one octahedrally and one tetrahedrally coordinated aluminum atom was confirmed (Fig. 6), and the $\mathrm{C}_{1}$ isomer (Fig. 4) with the two ester groups occupying an equatorial and an axial position was found ("equatorial position" refers to the $\mathrm{Al}_{2}(\mu-\mathrm{OR})_{2}$ plane). Selected bond distances and angles are given in Table 2.

The Al-O distances of the chelating tbuac are longer for the "ester" oxygen compared to that of the "keto" oxygen.
Fig. 6 Molecular structure of $\left[\mathrm{Al}\left(\mathrm{O}^{\mathrm{i}} \mathrm{Pr}\right)_{2}\left({ }^{\mathrm{t}} \mathrm{buac}\right)\right]_{2}$ in the crystalline state

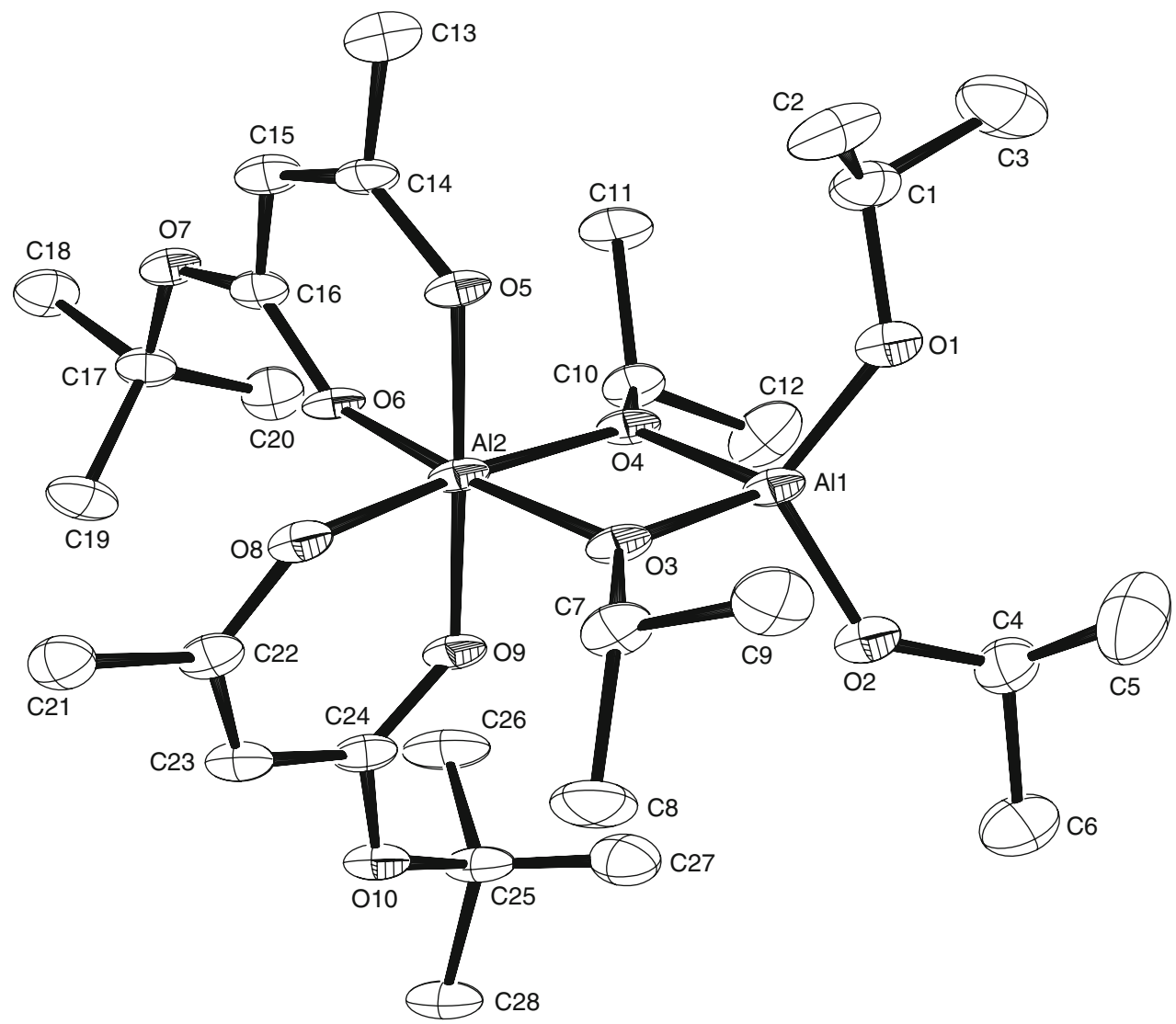


Table 2 Selected bond distances $[\mathrm{pm}]$ and angles [deg] of $\left[\mathrm{Al}\left(\mathrm{O}^{\mathrm{i}} \mathrm{Pr}\right)_{2}\left({ }^{\mathrm{t}} \text { buac }\right)\right]_{2}$

\begin{tabular}{lrlr}
\hline $\mathrm{Al}(1)-\mathrm{O}(1)$ & $171.4(5)$ & $\mathrm{Al}(2)-\mathrm{O}(6)$ & $190.4(4)$ \\
$\mathrm{Al}(1)-\mathrm{O}(2)$ & $171.5(4)$ & $\mathrm{Al}(2)-\mathrm{O}(8)$ & $185.0(4)$ \\
$\mathrm{Al}(1)-\mathrm{O}(3)$ & $180.4(4)$ & $\mathrm{Al}(2)-\mathrm{O}(9)$ & $192.6(4)$ \\
$\mathrm{Al}(1)-\mathrm{O}(4)$ & $180.0(4)$ & $\mathrm{O}(5)-\mathrm{C}(14)$ & $128.1(6)$ \\
$\mathrm{Al}(2)-\mathrm{O}(3)$ & $190.1(4)$ & $\mathrm{O}(6)-\mathrm{C}(16)$ & $126.9(6)$ \\
$\mathrm{Al}(2)-\mathrm{O}(4)$ & $191.7(4)$ & $\mathrm{O}(8)-\mathrm{C}(22)$ & $129.7(7)$ \\
$\mathrm{Al}(2)-\mathrm{O}(5)$ & $187.2(4)$ & $\mathrm{O}(9)-\mathrm{C}(24)$ & $125.7(7)$ \\
$\mathrm{O}(1)-\mathrm{Al}(1)-\mathrm{O}(2)$ & $113.5(2)$ & $\mathrm{O}(4)-\mathrm{Al}(2)-\mathrm{O}(6)$ & $93.9(2)$ \\
$\mathrm{O}(3)-\mathrm{Al}(1)-\mathrm{O}(4)$ & $113.2(2)$ & $\mathrm{O}(4)-\mathrm{Al}(2)-\mathrm{O}(9)$ & $87.4(2)$ \\
$\mathrm{Al}(1)-\mathrm{O}(3)-\mathrm{Al}(2)$ & $100.4(2)$ & $\mathrm{O}(5)-\mathrm{Al}(2)-\mathrm{O}(6)$ & $91.0(2)$ \\
$\mathrm{Al}(1)-\mathrm{O}(4)-\mathrm{Al}(2)$ & $99.9(2)$ & $\mathrm{O}(5)-\mathrm{Al}(2)-\mathrm{O}(8)$ & $89.4(2)$ \\
$\mathrm{O}(3)-\mathrm{Al}(2)-\mathrm{O}(4)$ & $77.1(2)$ & $\mathrm{O}(6)-\mathrm{Al}(2)-\mathrm{O}(8)$ & $92.9(2)$ \\
$\mathrm{O}(3)-\mathrm{Al}(2)-\mathrm{O}(5)$ & $89.9(2)$ & $\mathrm{O}(6)-\mathrm{Al}(2)-\mathrm{O}(9)$ & $86.0(2)$ \\
$\mathrm{O}(3)-\mathrm{Al}(2)-\mathrm{O}(8)$ & $96.2(2)$ & $\mathrm{O}(8)-\mathrm{Al}(2)-\mathrm{O}(9)$ & $90.8(2)$ \\
$\mathrm{O}(3)-\mathrm{Al}(2)-\mathrm{O}(9)$ & $93.1(2)$ & $\mathrm{O}(3)-\mathrm{Al}(2)-\mathrm{O}(6)$ & $170.9(2)$ \\
$\mathrm{O}(4)-\mathrm{Al}(2)-\mathrm{O}(5)$ & $92.8(2)$ & $\mathrm{O}(4)-\mathrm{Al}(2)-\mathrm{O}(8)$ & $172.9(2)$ \\
& & $\mathrm{O}(5)-\mathrm{Al}(2)-\mathrm{O}(9)$ & $177.0(2)$ \\
\hline
\end{tabular}

An additional trans effect from the bridging alkoxo groups causes shorter bond lengths for the equatorial bonds. The Al-O bond distances of the tbuac ligands decrease in the order Al-O $\mathrm{O}_{\text {ester,ax }}(192.6(4) \mathrm{pm})>\mathrm{Al}-\mathrm{O}_{\text {ester,eq }}(190.4(4)$ pm) $>\mathrm{Al}-\mathrm{O}_{\text {keto,ax }}(187.2(4) \mathrm{pm})>\mathrm{Al}-\mathrm{O}_{\text {keto,eq }}(185.0(4)$ pm). Vice versa, the different substituents trans to the bridging alkoxo groups result in an asymmetric bridging situation $(\mathrm{Al}(2)-\mathrm{O}(3) \quad 190.1(4) \mathrm{pm}$ and $\mathrm{Al}(2)-\mathrm{O}(4)$ 191.7(4) pm).

The bridging $\mathrm{Al}-\mathrm{O}$ distances $\mathrm{Al}(2)-\mathrm{O}(3)$ and $\mathrm{Al}(2)-$ $\mathrm{O}(4)$ are significantly longer than $\mathrm{Al}(1)-\mathrm{O}(3)$ (180.4(4) pm) and $\mathrm{Al}(1)-\mathrm{O}(4)$ (180.0(4) pm), showing the bond distances from the bridging oxygen atoms to the octahedral aluminum center to be significantly longer than to the tetrahedral center. As expected, the distances $\mathrm{Al}(1)-\mathrm{O}(1)$ 171.4(5) and $\mathrm{Al}(1)-\mathrm{O}(2)$ 171.5(4) of the terminal alkoxo groups are distinctly shorter than that of the bridging groups.

For a better understanding of the coordination behavior of the $\beta$-ketoesterate ligands and their above mentioned formation at room temperature, the trisubstituted monomeric $\mathrm{Al}(\beta \text {-ketoesterate })_{3}$ complexes were also studied for all ligands. The complexes were prepared by addition of three molar equivalents of the $\beta$-ketoester to the alkoxide. The structure of $\mathrm{Al}^{\mathrm{t}}$ buac) $)_{3}$ was previously determined [13]. The ${ }^{27} \mathrm{Al}$ chemical shift of $4.78 \mathrm{ppm}$ in the NMR spectrum of $\mathrm{Al}(\mathrm{etac})_{3}$ is in line with the octahedral coordination of the aluminum atom. ${ }^{1} \mathrm{H}$ and ${ }^{13} \mathrm{C}$ NMR spectra of the $\operatorname{Al}(\beta \text {-ketoesterate })_{3}$ compounds showed up to 4 signal sets for the ester groups. This is a result of the coexistence of different isomers in solution, viz. a $\mathrm{C}_{3}$ symmetric isomer with the keto and ester oxygen atoms
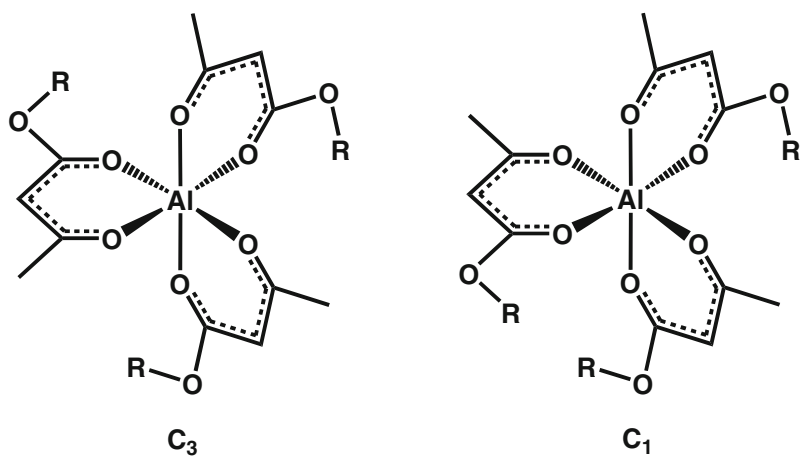

Fig. 7 Different isomers of $\mathrm{Al}(\beta \text {-ketoesterate })_{3}$ complexes

respectively in fac arrangement and a $\mathrm{C}_{1}$ symmetric isomer with mer arrangement. In the $\mathrm{C}_{3}$ symmetric complex, all ligands are symmetrically equivalent giving rise to one set of signals in the NMR spectra, whereas for the $C_{1}$ symmetric complex all ligands are non-equivalent, causing one set of signals for each ligand (Fig. 7). As a matter of fact each isomer forms a pair of enantiomers, which in the context of the NMR studies has no further consequences. Although the signals could not be assigned to the $\mathrm{C}_{1}$ or $\mathrm{C}_{3}$ isomer, the observation of four signal sets of equal intensity in the ${ }^{1} \mathrm{H}$ NMR spectra corresponds to a 1:3 ratio of $\mathrm{C}_{3}$ and $\mathrm{C}_{1}$ symmetric species. Variable temperature NMR showed that the isomers can transform into each other. Coalescence was observed above $80{ }^{\circ} \mathrm{C}$, causing an averaged signal set (Fig. 8). EXSY experiments confirmed exchange of the $\beta$-ketoesterate signals between the different isomers. The $\mathrm{CH}_{3}$ region of the EXSY spectrum of $\mathrm{Al}(\mathrm{etac})_{3}$ is given in Fig. 9, showing exchange for the $\mathrm{CH}_{3} \mathrm{CO}(1.90-1.80 \mathrm{ppm})$ and $\mathrm{OCH}_{2} \mathrm{CH}_{3}(1.12-0.92$ ppm) signals.

The ${ }^{1} \mathrm{H}$ chemical shifts of the $\beta$-keto protons of the coordinated ligands for the mono- and trisubstituted complexes are compared in Table 3. The signals for the trisubstituted species are shifted to somewhat higher ppm values for all complexes (5.18-5.08 ppm compared to $5.10-4.98 \mathrm{ppm}$ for $\left.\left[\mathrm{Al}(\mathrm{OR})_{2}(\beta \text {-ketoesterate })\right]_{2}\right)$. No correlation between the chemical shifts of $\beta$-keto protons and carbons and the size of the ester OR groups was observed. The ${ }^{13} \mathrm{C}$ NMR shift differences between the mono- and trisubstituted complexes were not significant.

It is known that transesterification can occur as a possible side reaction during the reaction of metal alkoxides with esters [8] or can be used selectively as a preparative synthetic method [14]. Transesterification would change the structural and chemical properties of the metal alkoxides; for $\beta$-ketoesters with a functional OR group this would also result in the loss of the organic functionality. Transesterification as possible side reaction was studied for the reaction of the $\beta$-ketoesters with $\left[\mathrm{Al}\left(\mathrm{O}^{\mathrm{i}} \mathrm{Pr}\right)_{3}\right]_{4}$ by NMR spectroscopy. If transesterification would occur, iso-propyl acetoacetate derivatives would be formed. ${ }^{1} \mathrm{H}$ NMR 


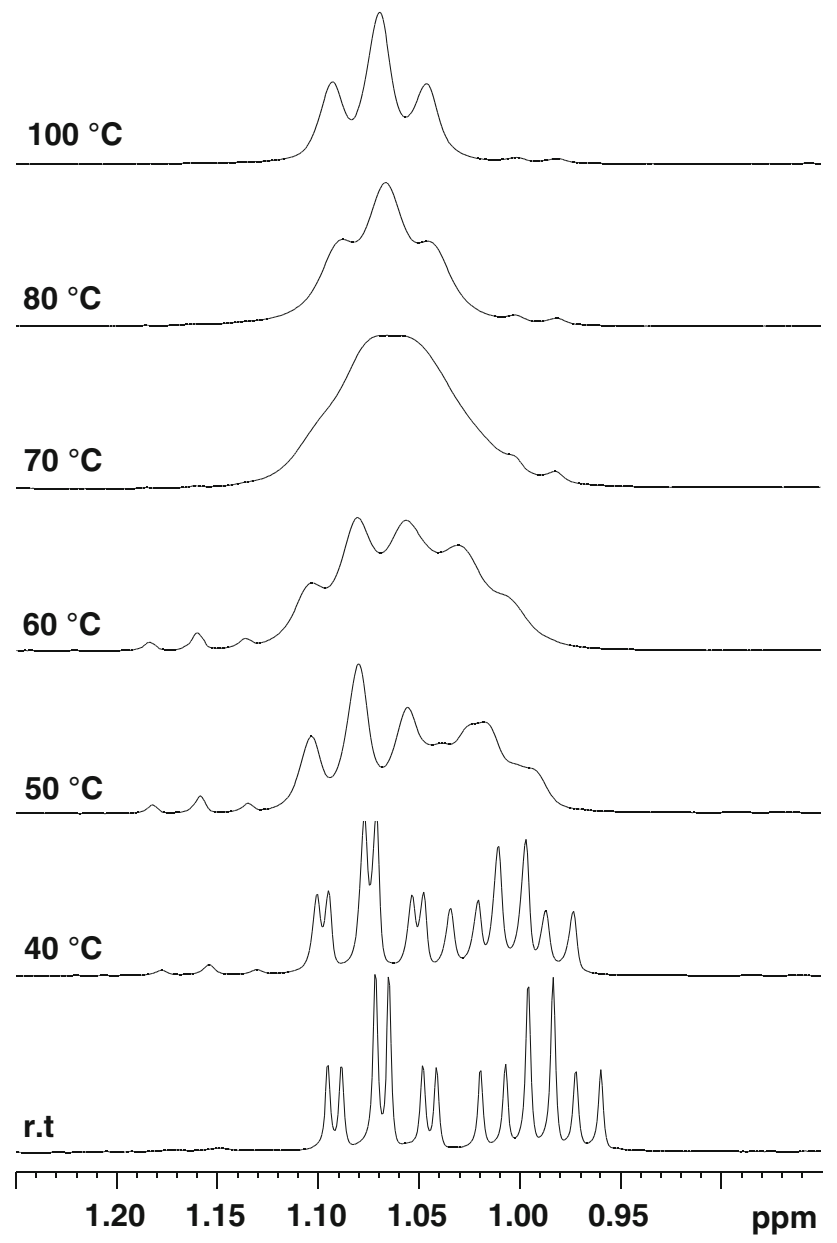

Fig. 8 Temperature dependent ${ }^{1} \mathrm{H}$ NMR $\mathrm{OCH}_{2} \mathrm{CH}_{3}$ resonances of $\mathrm{Al}(\mathrm{etac})_{3}\left(\mathrm{~d}_{8}\right.$-toluene $)$

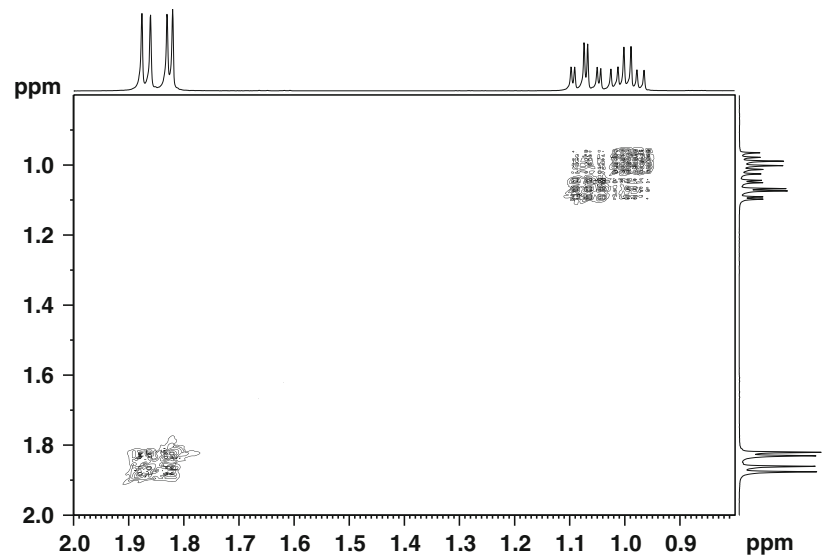

Fig. 9 EXSY spectrum of $\mathrm{Al}(\text { etac })_{3}$ at $20{ }^{\circ} \mathrm{C}$ in $\mathrm{d}_{8}$-toluene, $\mathrm{CH}_{3^{-}}$ region

spectroscopy allows easy monitoring of the transesterification because the signal of the methine proton of the formed iso-propylacetoacetate at $5.15-4.85 \mathrm{ppm}$ (in the monosubstituted complex) and $5.35 \mathrm{ppm}$ (in the trisubstituted complex) is considerably shifted to higher ppm
Table 3 Comparison of ${ }^{1} \mathrm{H}$ NMR shifts of $\beta$-keto protons of $\left[\mathrm{Al}\left(\mathrm{O}^{\mathrm{i}} \mathrm{Pr}\right)_{2}(\beta \text {-ketoesterate })\right]_{2}$ and $\mathrm{Al}(\beta \text {-ketoesterate })_{3}$ complexes (in $\mathrm{C}_{6} \mathrm{D}_{6}$ )

\begin{tabular}{lll}
\hline & {$\left[\mathrm{Al}\left(\mathrm{O}^{\mathrm{i}} \operatorname{Pr}\right)_{2}(\beta \text {-ketoesterate })\right]_{2}$} & $\mathrm{Al}(\beta \text {-ketoesterate })_{3}$ \\
\hline meac-H & $5.07(5.06)^{\mathrm{a}}$ & 5.17 \\
etac-H & $5.10(5.08)^{\mathrm{a}}$ & $5.18 / 5.17$ \\
${ }^{\mathrm{i}}$ prac-H & $5.06(5.04)^{\mathrm{a}}$ & 5.16 \\
tbuac-H & $5.06 / 5.05 / 4.98$ & $5.13 / 5.10 / 5.08$ \\
aaa-H & 5.10 & 5.15 \\
meaa-H & 5.05 & 5.14 \\
\hline
\end{tabular}

$\overline{{ }^{a} \text { Values in brackets: signals of minor components with different }}$ coordination geometry

values compared with the signals of all the other esters. Additional evidence was obtained from HMBC experiments which show long range coupling between the methine proton and a carbonyl carbon if the iso-propyl ester was formed.

In no case was transesterification observed, neither at room temperature nor at $120{ }^{\circ} \mathrm{C}$, except for the reaction of $\left[\mathrm{Al}\left(\mathrm{O}^{\mathrm{i}} \mathrm{Pr}\right)_{3}\right]_{4}$ with three equivalents of ${ }^{\mathrm{t}}$ buac- $\mathrm{H}$ at $120^{\circ} \mathrm{C}$. $\mathrm{Al}\left({ }^{\mathrm{t}} \text { buac }\right)_{3}$, formed at room temperature, reacts at $120^{\circ} \mathrm{C}$ overnight with the liberated iso-propanol still present in the reaction solution to give $\mathrm{Al}\left({ }^{\mathrm{t}} \mathrm{buac}\right)_{3-x}\left({ }^{\mathrm{i}} \mathrm{prac}\right)_{x}$. This reaction was studied in more detail by NMR spectroscopy, recording spectra in situ at $80^{\circ} \mathrm{C}$ in intervals of 2 and $4 \mathrm{~h}$, respectively. Figure 10 shows the time dependence of the ratio between free iso-propanol and tert-butanol, representing the degree of transesterification, since free tertbutanol only could originate from the transesterification reaction. Longer reaction times finally led to complete substitution of all tert-butoxo groups by iso-propanol, giving $\mathrm{Al}\left({ }^{\mathrm{i}} \mathrm{prac}\right)_{3}$. Interestingly, for the reaction of $\left[\mathrm{Al}\left(\mathrm{O}^{\mathrm{i}} \mathrm{Pr}\right)_{3}\right]_{4}$ with one equivalent of ${ }^{\mathrm{t}}$ buac- $\mathrm{H}$, after heating for $18 \mathrm{~h}$ to $120^{\circ} \mathrm{C}$, no transesterification was observed and crystalline $\left[\mathrm{Al}\left(\mathrm{O}^{\mathrm{i}} \mathrm{Pr}\right)_{2}\left({ }^{\mathrm{t}} \mathrm{buac}\right)\right]_{2}$ was the only product. From the results it is assumed that the steric demand of the

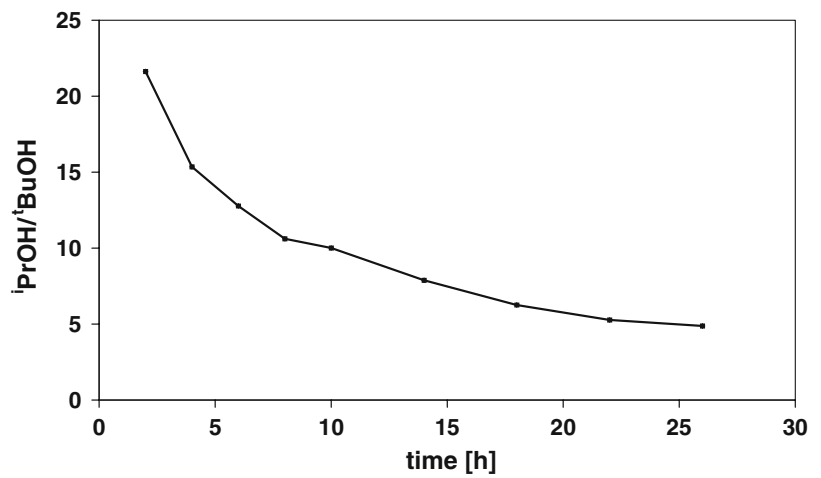

Fig. 10 Time dependence of ${ }^{i} \mathrm{PrOH} /{ }^{t} \mathrm{BuOH}$ ratio at $80^{\circ} \mathrm{C}$ in $\mathrm{d}_{8}$-toluene 
tert-butoxo group in $\mathrm{Al}\left({ }^{\mathrm{t}} \text { buac) }\right)_{3}$ favors transesterification and formation of sterically less demanding $\mathrm{Al}\left({ }^{\mathrm{t}} \mathrm{buac}\right)_{3-}$ ${ }_{x}\left({ }^{\mathrm{i}} \text { prac }\right)_{x}$ species. Since one ${ }^{\mathrm{t}}$ buac ligand in $\left[\mathrm{Al}\left(\mathrm{O}^{\mathrm{i}} \mathrm{Pr}\right)_{2}\right.$ ('buac) $]_{2}$ is replaced by two iso-propoxo groups, the steric constraint might be low enough to not cause transesterification in this case.

\section{Conclusions}

The substitution of $\left[\mathrm{Al}\left(\mathrm{O}^{\mathrm{i}} \mathrm{Pr}\right)_{3}\right]_{4}$ with one or three equivalents of the $\beta$-ketoesters meac- $\mathrm{H}$, etac- $\mathrm{H}$, ${ }^{\mathrm{i}}$ prac- $\mathrm{H}$, ${ }^{\mathrm{t}}$ buac- $\mathrm{H}$, aaa- $\mathrm{H}$ and meaa- $\mathrm{H}$ per $\mathrm{Al}$ resulted in derivatives $\left[\mathrm{Al}\left(\mathrm{O}^{\mathrm{i}} \mathrm{Pr}\right)_{2}\right.$ $(\beta$-ketoesterate $)]_{2}$ and $\mathrm{Al}(\beta \text {-ketoesterate })_{3}$, respectively, although special synthesis protocols are required to obtain $\left[\mathrm{Al}\left(\mathrm{O}^{\mathrm{i}} \mathrm{Pr}\right)_{2}(\beta \text {-ketoesterate })\right]_{2}$ derivatives. The monosubstituted derivatives $\left[\mathrm{Al}\left(\mathrm{O}^{\mathrm{i}} \mathrm{Pr}\right)_{2}(\beta \text {-ketoesterate })\right]_{2}$ are asymmetric dimers with one octahedrally coordinated $\mathrm{Al}$ center substituted by two ester ligands and one tetrahedrally coordinated $\mathrm{Al}$ atom surrounded by two bridging and two terminal iso-propoxo groups. The trisubstituted compounds $\operatorname{Al}(\beta \text {-ketoesterate })_{3}$ are mononuclear octahedral complexes, giving rise to $\mathrm{C}_{1}$ and $\mathrm{C}_{3}$ symmetric isomers due to the asymmetric chelating ligand.

The alkoxo group of the esters had no influence on the structure of the products and only minor influence on the electronic properties, reflected in slightly different ${ }^{1} \mathrm{H}$ NMR shifts of the bridging and terminal iso-propoxo groups of the monosubstituted dimers.

Only in two cases the type of ligand used had consequences on the preparation and stability of the substituted aluminum alkoxide derivatives: (i) The complex $\mathrm{Al}\left({ }^{\mathrm{t}}\right.$ buac) ${ }_{3}$ undergoes transesterification in the presence of iso-propanol, giving $\mathrm{Al}\left({ }^{\mathrm{i}} \mathrm{prac}\right)_{x}\left({ }^{\mathrm{t}} \mathrm{buac}\right)_{3-x}$. This behavior is attributed to the steric bulk of the tert-butoxo groups. (ii) Due to the thermal reactivity of the methacrylate substituent of meaa$\mathrm{H}$, direct reaction of $\left[\mathrm{Al}\left(\mathrm{O}^{\mathrm{i}} \mathrm{Pr}\right)_{3}\right]_{4}$ with meaa- $\mathrm{H}$ is not possible. However, $\left[\mathrm{Al}\left(\mathrm{O}^{\mathrm{i}} \mathrm{Pr}\right)_{2}(\mathrm{meaa})\right]_{2}$ can be prepared by thermal de-oligomerization of $\left[\mathrm{Al}\left(\mathrm{O}^{\mathrm{i}} \mathrm{Pr}\right)_{3}\right]_{4}$ followed by addition of the ligand at room temperature.

Contrary to analogous reactions with acetylacetone [5, $6]$, the monosubstituted complexes $\left[\mathrm{Al}\left(\mathrm{O}^{\mathrm{i}} \mathrm{Pr}\right)_{2}(\beta\right.$-ketoesterate) $]_{2}$ were not formed when the compounds are reacted at room temperature. Mixtures of trisubstituted $\mathrm{Al}(\beta$-ketoesterate $)_{3}$ and non-reacted $\left[\mathrm{Al}\left(\mathrm{O}^{\mathrm{i}} \mathrm{Pr}\right)_{3}\right]_{4}$ were instead obtained. Formation of the monosubstituted complexes $\left[\mathrm{Al}\left(\mathrm{O}^{\mathrm{i}} \mathrm{Pr}\right)_{2}(\beta \text {-ketoesterate })\right]_{2}$ was achieved by heating this mixture at $120{ }^{\circ} \mathrm{C}$, and they are stable after cooling to room temperature, indicating $\left[\mathrm{Al}\left(\mathrm{O}^{\mathrm{i}} \mathrm{Pr}\right)_{2}(\beta \text {-ketoesterate })\right]_{2}$ has a higher thermodynamic stability. Compared to $\left[\mathrm{Al}\left(\mathrm{O}^{\mathrm{i}} \mathrm{Pr}\right)_{2}\right.$ $(\mathrm{acac})]_{2}[5]$, no aging to trimeric $\left[\mathrm{Al}\left(\mathrm{O}^{\mathrm{i}} \mathrm{Pr}\right)_{2}(\beta \text {-diketonate })\right]_{3}$ was observed for the $\beta$-ketoesterate derivatives. Thus, significant differences in reactivity of $\beta$-ketoesters and $\beta$-diketones with $\left[\mathrm{Al}\left(\mathrm{O}^{\mathrm{i}} \mathrm{Pr}\right)_{3}\right]_{4}$ are noticed, since the structures of the formed derivatives may be different depending on the side conditions (temperature, time). This may have important consequences if those ligands are used to modify aluminum alkoxides for sol-gel processing.

\section{X-Ray Structure Analysis}

A plate-like crystal of $\left[\mathrm{Al}\left(\mathrm{O}^{\mathrm{i}} \mathrm{Pr}\right)_{2}{ }^{\mathrm{t}}\right.$ buac $\left.)\right]_{2}$ was mounted on a Siemens SMART diffractometer with an area detector and measured in a nitrogen stream. Mo- $\mathrm{K}_{\alpha}$ radiation ( $\lambda=71.069 \mathrm{pm}$, graphite monochromator) was used for all measurements. The data collection (Table 4) at $100 \mathrm{~K}$ covered a hemisphere of the reciprocal space, by a combination of three sets of exposures. Each set had a different $\phi$ angle for the crystal, and each exposure took $25 \mathrm{sec}$ and covered $0.3^{\circ}$ in $\omega$. The crystal-to-detector distance was $5 \mathrm{~cm}$. The crystal partially decomposed during measurement. The data were corrected for polarization and Lorentz effects, and an empirical absorption correction (SADABS) was employed. The cell dimensions were refined with all unique reflections.

The structure was solved by direct methods (SHELXS97). Refinement was performed by the full-matrix leastsquares method based on $F^{2}$ (SHELXL97) with anisotropic

Table 4 Crystallographic and structural parameters of $\left[\mathrm{Al}\left(\mathrm{O}^{\mathrm{i}} \mathrm{Pr}\right)_{2}\right.$ (tbuac) $]_{2}$

\begin{tabular}{|c|c|}
\hline Empirical formula & $\mathrm{C}_{28} \mathrm{H}_{54} \mathrm{Al}_{2} \mathrm{O}_{10}$ \\
\hline Formula weight & 604.7 \\
\hline Crystal system & Monoclinic \\
\hline Space group & $P 2_{1} / n$ \\
\hline$a[\mathrm{pm}]$ & $9.553(4)$ \\
\hline$b[\mathrm{pm}]$ & $18.029(7)$ \\
\hline$c[\mathrm{pm}]$ & $20.475(9)$ \\
\hline$\alpha\left[^{\circ}\right]$ & 90.00 \\
\hline$\beta\left[^{\circ}\right]$ & $91.825(8)$ \\
\hline$\gamma\left[^{\circ}\right]$ & 90.00 \\
\hline Volume $\left[10^{6} \mathrm{pm}^{3}\right]$ & $3525(3)$ \\
\hline$Z$ & 4 \\
\hline Calcd. density $\left[\mathrm{g} \mathrm{cm}^{-3}\right]$ & 1.139 \\
\hline$\mu\left[\mathrm{mm}^{-1}\right]$ & 0.129 \\
\hline Crystal size $[\mathrm{mm}]$ & $1.60 \times 1.40 \times 0.10$ \\
\hline $2 \theta$ range $\left[{ }^{\circ}\right]$ & $2.41-28.45$ \\
\hline Reflections collected/unique & $12759 / 6606$ \\
\hline Data/parameters & $6606 / 377$ \\
\hline GOF on $F^{2}$ & 0.991 \\
\hline$R[I>2 \sigma(I)]$ & 0.1165 \\
\hline $\mathrm{w} R_{2}$ & 0.3253 \\
\hline Largest peak/hole $\left[\mathrm{e} \AA^{-3}\right]$ & $0.581 /-0.375$ \\
\hline
\end{tabular}


thermal parameters for all non-hydrogen atoms. Hydrogen atoms were inserted in calculated positions and refined riding with the corresponding atom.

CCDC-694178 contains the supplementary crystallographic data for this paper. These data can be obtained free of charge from The Cambridge Crystallographic Data Center via www.ccdc.cam.ac.uk/data_request/cif.

Acknowledgment This work was supported by the Fonds zur Förderung der wissenschaftlichen Forschung (FWF), Austria.

Open Access This article is distributed under the terms of the Creative Commons Attribution Noncommercial License which permits any noncommercial use, distribution, and reproduction in any medium, provided the original author(s) and source are credited.

\section{References}

1. Livage J, Henry M, Sanchez C (1988) Prog Solid State Chem $18: 259$

2. Turova NY, Kozunov VA, Yanovskii AI, Bokii NG, Struchkov YT, Tarnopol'skii BL (1979) J Inorg Nuc Chem 41:5
3. Abraham A, Prins R, van Bokhoven JA, van Eck ERH, Kentgens APM (2006) J Phys Chem B 110:6553

4. Cayton RH, Chisholm MH, Davidson ER, DiStasi VF, Du P, Huffman JC (1991) Inorg Chem 30:1020

5. Wengrovius JH, Garbauskas MF, Williams EA, Goint RC, Donahue PE, Smith JF (1986) J Am Chem Soc 108:982

6. Garbauskas MF, Wengrovius JH (1987) Acta Crystallogr Sect C Cryst Struct Commun C43:2441

7. Schubert U (2007) Acc Chem Res 40:730 and references cited therein

8. Ivanovici S, Puchberger M, Fric H, Kickelbick G (2007) Monatsh Chem 138:529

9. Cauro-Gamet LC, Hubert-Pfalzgraf LG, Lecocq S (2004) Z Anorg Allg Chem 630:2071

10. Shiner VJ Jr, Whittaker D, Vernandez VP (1963) J Am Chem Soc $85: 2318$

11. Kleinschmidt DC, Shiner VJ Jr, Whittaker D (1973) J Org Chem 38:3334

12. Kriz O, Casensky B, Lycka A, Fusek J, Hermanek S (1984) J Magn Reson 60:375

13. Dharmaprakash MS, Thamotharan D, Neelgund GM, Shivashankar SA (2006) Acta Cryst E62:m434

14. Pajot N, Papiernik R, Hubert-Pfalzgraf LG, Vaissermann J, Parraud S (1995) J Chem Soc Chem Commun 1817 